\title{
Arnold Diffusion in the Swing Equations of a Power System
}

\author{
FATHI M. A. SALAM, MEMBER, IEEE, JERROLD E. MARSDEN, AND PRAVIN P. VARAIYA, FELLOW, IEEE
}

\begin{abstract}
We present an application of the theory of Arnold diffusion to interconnected power systems. Using a Hamiltonian formulation, we show that Arnold diffusion arises on certain energy levels of the swing equations model. The occurrence of Arnold diffusion entails complex nonperiodic dynamics and erratic transfer of energy between the subsystems. Conditions under which Arnold diffusion exists in the dynamics of the swing equations are found by using the vector-Melnikov method. These conditions become analytically explicit in the case when some of the subsystems undergo relatively small oscillations. Perturbation and parameter regions are found for which Arnold diffusion occurs. These regions allow for a class of interesting systems from the point of view of power systems engineering.
\end{abstract}

\section{INTRODUCTION}

W E APPLY the results on Arnold diffusion of Holmes and Marsden [26] (see also [1, sect. 4]) to power systems [45]. We note that the results in fact apply to all systems of the forced pendulum family such as interconnected power systems employing the swing equations model, coupled Josephson junction circuits with negligible dissipation, a Josephson junction driven by a dircet current source (plus a small alternating current) coupled to two (respectively one) nonlinear oscillators, and coupled mechanical pendulums. The precise calculations are carried out here for a dynamical model of interconnected power systems.

In the dynamical behavior of a large interconnected power system, the question of transient stability is often considered. This concerns the system's behavior following a sudden fault (such as short circuit) or a large impact (such as lightning). The transient stability is precisely the Lyapunov stability in a state-space formulation of a simplified differential equations model possessing multiple equilibria. Let the dynamics be given by $\dot{x}=f(x)$ and let $x_{0}$ be a stable equilibrium point which is presumably "closest" to the prefault equilibrium point (see [32], [9]). The transient stability problem is to determine whether a given point in the state space belongs to the region of stability of this stable equilibrium point. Thus the transient stability problem leads to an investigation of the region of stability of a given stable equilibrium point [28], [33], [13],

Manuscript received March 14, 1984. This work was supported in part by DOE Contracts DE-AT03-82 ER 12097 and DE-AS01-78ER29135, and by the National Science Foundation under Grant ECS-811213.

F. M. A. Salam was with the University of California, Berkeley, CA 94720 . He is now with the Department of Mechanical Engineering and Mechanics, Drexel University, Philadelphia, PA.

J. E. Marsden and P. P. Varaiya are with the Department of Electrical Engineering and Computer Sciences, University of California, Berkeley, CA 94720 .
[14], [18]. Many studies of transient stability [28], [33], [13], [14], [18] have been conducted exploiting a first integral of the differential equation as a Lyapunov (energy) function.

Kopell and Washburn [29] were the first to show the presence of chaotic motion in the classical swing equations model of power systems for a 2-degree-of-freedom system (3 generators). Their work is based on the original Melnikov method for vector fields (see Holmes [24]) and the energy function was not exploited to locate the energy levels where chaos resides.

Here we show the presence of Arnold diffusion in the $(n \geqslant 3)$-degree-of-freedom Hamiltonian system (with constraints) of the classical model. In the case when $(n=2)$ only horseshoes are present. This case is analogous to the one obtained by Kopell and Washburn except that we also specify the energy levels on which chaos resides, an advantage of exploiting the energy function.

The paper is organized in the following way. In Section II we summarize the key result of Holmes and Marsden [26]. Section III contains some motivation and the derivation of the swing equations model. In Section IV we consider specific choices of parameter ranges to simplify the model before applying the results of Section II. In this section we also study the Hamiltonian formulation of the swing equations. They form a $2 n$ degree of freedom system with two time-independent constraints. In Section $V$ we show that the conditions of Section II can be satisfied for a large choice of parameters. These conditions can be simplified if all but one of the subsystems undergo small oscillations. This case is discussed in Section VI. Conclusions and suggestions for future work are collected in Section VII.

\section{ARNOLd DiF̀FUSION IN HAMILTONIAN SYSTEMS}

In this section we summarize the results of Holmes and Marsden [26] for Hamiltonian systems with $n$-degrees of freedom $(n \geqslant 3)$. These results extend the work of Arnold [11], for more discussion of Arnold diffusion, see [1].

\section{Problem Statement}

Consider the unperturbed Hamiltonian system

$$
H^{0}(q, p, \vec{x}, \vec{y})=F(q, p)+G(\vec{x}, \vec{y})
$$

where $F$ is a IIamiltonian which possesses a homoclinic orbit $(\bar{q}, \bar{p})$ associated with a hyperbolic saddle point $q_{0}, p_{0}$. Let $\bar{h}$ be the energy constant of this orbit, i.e., $F(\bar{q}, \bar{p})=\bar{h}$. The parameters $(q, p, \vec{x}, \vec{y})$ are assumed to be 
canonical coordinates on a $2(n+1)$-dimensional symplectic manifold $\boldsymbol{P} ; q$ and $p$ are real and $\vec{x}=\left(x_{1}, \cdots, x_{n}\right), \vec{y}=$ $\left(y_{1}, \cdots, y_{n}\right)$ are $n$-vectors. We assume that in a certain region of the state space a canonical transformation to action-angle coordinates $\left(\vartheta_{1}, \cdots, \vartheta_{n}, I_{1}, \cdots, I_{n}\right)$ can be found such that the system (2.1) takes the form

$$
H^{0}(q, p, \vec{\vartheta}, \vec{I})=F(q, p)+\sum_{i=1}^{n} G_{i}\left(I_{i}\right)
$$

where

$$
G_{j}(0)=0, \quad \text { for all } j
$$

and

$$
\Omega_{j}\left(I_{j}\right)=\frac{\partial G_{j}}{\partial I_{j}}>0, \quad \text { for } I_{j}>0 .
$$

Applying the reduction procedure (see Holmes and Marsden [25], [26]), we solve $H^{0}=h$ for $I_{n}$, thereby eliminating the action $I_{n}$. We also replace the time variable by the $2 \pi$-periodic angle $\vartheta_{n}$. Then the equations

$$
\begin{aligned}
G_{j}\left(I_{j}\right) & =h_{j} \\
\vartheta_{j} & =\Omega_{j}\left(I_{j}\right) \vartheta_{n}+\vartheta_{j}(0), \quad j=1, \cdots, n-1 \\
q & =q_{0}, \quad p=p_{0}
\end{aligned}
$$

describe an $(n-1)$-parameter family of invariant $(n-1)$ dimensional tori $\boldsymbol{T}\left(h_{1}, \cdots, h_{n-1}\right)$. For a fixed set of $h_{1}, \cdots, h_{n-1}$, the torus $\boldsymbol{T}\left(h_{1}, \cdots, h_{n-1}\right)$ is connected to itself by the $n$-dimensional homoclinic manifold

$$
\begin{aligned}
G_{j}\left(I_{j}\right) & =h_{j} \\
\vartheta_{j} & =\Omega_{j}\left(I_{j}\right) \vartheta_{n}+\vartheta_{j}^{0}, \quad \\
q & =\bar{q}\left(\vartheta_{n}-\vartheta_{n}^{0}\right), \quad p=\bar{p}\left(\vartheta_{n}-\vartheta_{n}^{0}\right) .
\end{aligned}
$$

This manifold consists of the coincident stable and unstable manifolds of the torus $\boldsymbol{T}\left(h_{1}, \cdots, h_{n-1}\right)$, i.c.,

$$
\boldsymbol{W}^{s}\left(\boldsymbol{T}\left(h_{1}, \cdots, h_{n-1}\right)\right)=\boldsymbol{W}^{u}\left(\boldsymbol{T}\left(h_{1}, \cdots, h_{n-1}\right)\right) .
$$

The perturbed problem considered here has the following form

$$
H^{\mu}(q, p, \vec{\vartheta}, \vec{I})=F(q, p)+\sum_{i=1}^{n} G_{i}\left(I_{i}\right)+\mu H^{1}(q, p, \vec{\vartheta}, \vec{I})
$$

where $H^{\mathbf{1}}$ is $2 \pi$-periodic in $\vartheta_{1}, \cdots, \vartheta_{n}$ (precisely, $2 \pi / \Omega_{i}\left(I_{i}\right)$-periodic) and $\mu>0$. For sufficiently small $\mu$, KAM theory asserts that (under nonresonance and nondegeneracy conditions given below) a positive measure of the $(n-1)$-dimensional tori $T\left(h_{1}, \cdots, h_{n-1}\right)$ persists (see Arnold [12, appendix 8]). We denote these tori by $T_{\mu}\left(h_{1}, \cdots, h_{n-1}\right)$. Their corresponding stable and unstable manifolds $\boldsymbol{W}^{s}\left(\boldsymbol{T}_{\mu}\right)$, respectively, $\boldsymbol{W}^{u}\left(\boldsymbol{T}_{\mu}\right)$, are $C^{k}$ close, $k \geqslant$ 1 , to the unperturbed homoclinic manifold

$$
\boldsymbol{W}^{s}\left(\boldsymbol{T}\left(h_{1}, \cdots, h_{n-1}\right)\right)=\boldsymbol{W}^{u}\left(\boldsymbol{T}\left(h_{1}, \cdots, h_{n-1}\right)\right) .
$$

Let $h>\bar{h}$ be the total energy of the perturbed Hamiltonian $H^{\mu}$ of equation (2.6). Now consider the $n$-parameter family of orbits filling the unperturbed homoclinic mani- fold. Let

$$
\begin{aligned}
& \left(\bar{q}, \bar{p}, \vartheta_{1}, \cdots, \vartheta_{n}, I_{1}, \cdots, I_{n}\right) \\
& =\left(\bar{q}(t), \bar{p}(t), \Omega_{1}\left(I_{1}\right) t+\vartheta_{1}^{0}, \cdots, \Omega_{n}\left(I_{n}\right) t+\vartheta_{n}^{0}, I_{1}, \cdots, I_{n}\right)
\end{aligned}
$$

be the parameterization of these orbits and select one. Let $\left\{F, I^{1}\right\}$ denote the $(q, p)$ Poisson bracket of $F(q, p)$ and $H^{1}\left(q, p, \vartheta_{1}, \cdots, \vartheta_{n}, I_{1}, \cdots, I_{n}\right)$ evaluated on this orbit. Similarly let

$$
\left\{I_{k}, H^{1}\right\}=-\frac{\partial H^{1}}{\partial \vartheta_{k}}, \quad k=1, \cdots, n-1
$$

be evaluated on the same orbit. Then define the Melnikov vector $\boldsymbol{M}\left(\overrightarrow{\boldsymbol{\vartheta}}^{0}\right)=\left(\boldsymbol{M}_{1}, \cdots, \boldsymbol{M}_{n-1}, \boldsymbol{M}_{n}\right)$ by

$$
\begin{aligned}
\boldsymbol{M}_{k}\left(\vartheta_{1}^{0}, \cdots, \vartheta_{n}^{0}, h, h_{1}, \cdots, h_{n-1}\right) & :=\int_{-\infty}^{\infty}\left\{I_{k}, H^{1}\right\} d t, \\
k & =1, \cdots, n-1
\end{aligned}
$$

and

$$
\boldsymbol{M}_{n}\left(\vartheta_{1}^{0}, \cdots, \vartheta_{n}^{0}, h, h_{1}, \cdots, h_{n-1}\right):=\int_{-\infty}^{\infty}\left\{F, H^{1}\right\} d t
$$

where the integrals above are required to be, in an appropriate sense, conditionally convergent. That is, they mean

$$
\lim _{n \rightarrow \infty} \int_{-S_{n}}^{T_{n}}\{\cdots\} d t
$$

for suitable sequences $S_{n}, T_{n} \rightarrow \infty$.

Consider the following conditions:

(C1) $F$ possesses a homoclinic orbit $(\bar{q}(t), \bar{p}(t))$ connecting a saddle point $\left(q_{0}, p_{0}\right)$ to itself. Let $\bar{h}$ be the energy of this orbit.

(C2) $\Omega_{j}\left(I_{j}\right)=G_{j}^{\prime}\left(I_{j}\right)>0$ for $j=1, \cdots, n$.

(C3) The constants $G_{j}\left(I_{j}\right)=h_{j}, j=1, \cdots, n$ are chosen such that the unperturbed frequencies $\Omega_{1}\left(I_{1}\right), \cdots, \Omega_{n}\left(I_{n}\right)$ satisfy the nondegeneracy conditions (i.e., $\Omega_{j}^{\prime}\left(I_{j}\right) \neq 0, j=$ $1, \cdots, n)$ and the nonresonance condition, i.e.,

$$
\sum_{i=1}^{n} k_{i} \Omega_{i}\left(I_{i}\right)=0
$$

where $k_{i}$ are integers, implies $k_{i}=0$ for all $1 \leqslant i \leqslant n$.

(C4) The multiple $2 \pi$-periodic Melnikov vector $\boldsymbol{M}: R^{n}$ $\rightarrow R^{n}$ has at least one transversal zero, i.e., a point $\left(\vartheta_{1}^{\prime 0}, \cdots, \vartheta_{n}^{\prime 0}\right)$ such that

$$
\boldsymbol{M}\left(\boldsymbol{\vartheta}_{1}^{, 0}, \cdots, \boldsymbol{\vartheta}_{n}^{, 0}\right)=0
$$

and

$$
\operatorname{det}\left[\boldsymbol{D M}\left(\boldsymbol{\vartheta}_{1}^{, 0}, \cdots, \boldsymbol{\vartheta}_{n}^{, 0}\right)\right] \neq 0
$$

where $\boldsymbol{D M}$ is the $n \times n$ Jacobian matrix of the vector $\boldsymbol{M}$ with respect to the initial phases $\left(\vartheta_{1}^{0}, \cdots, \vartheta_{n}^{0}\right)$.

We can now state the main result.

\section{Theorem 2.1 (Holmes and Marsden)}

If conditions (C1)-(C4) hold for the perturbed system (2.6) then, for $\mu$ sufficiently small, the perturbed stable and unstable manifolds $W^{s}\left(T_{\mu}\right)$ and $W^{u}\left(T_{\mu}\right)$ of the perturbed torus $\boldsymbol{T}_{\mu}$ intersect transversally. Moreover, a finite transi- 
tion chain of such tori $\boldsymbol{T}_{\mu}^{1}, \cdots, \boldsymbol{T}_{\mu}^{k}$ can be chosen such that $\boldsymbol{W}^{u}\left(\boldsymbol{T}_{\mu}^{j}\right) \pitchfork \boldsymbol{W}^{s}\left(\boldsymbol{T}_{\mu}^{j+1}\right)$ and $\boldsymbol{W}^{u}\left(\boldsymbol{T}_{\mu}^{j+1}\right) \pitchfork \boldsymbol{W}^{s}\left(\boldsymbol{T}_{\mu}^{j}\right), 1 \leqslant j \leqslant k$ -1 .

( $\pitchfork$ denotes transversality of the intersection.) The transition chain of tori are responsible for the occurence of Arnold diffusion. Holmes and Marsden suggest that these transition tori can survive certain positive and negative damping, employing a technique which they had developed in [25].

An example which illustrates Theorem 2.1 is that of a simple pendulum linearly coupled to two nonlinear oscillators. Its perturbed Hamiltonian function can be written as follows (with the two oscillators in action-angle variables):

$$
\begin{aligned}
H^{\mu}= & \frac{p^{2}}{2}-\cos q+G_{1}\left(I_{1}\right)+G_{2}\left(I_{2}\right) \\
& +\left(\frac{\mu}{2}\right)\left[\left(\left(2 I_{1}\right)^{1 / 2} \sin \vartheta_{1}-q\right)^{2}+\left(\left(2 I_{2}\right)^{1 / 2} \sin \vartheta_{2}-q\right)^{2}\right] .
\end{aligned}
$$

One shows that conditions (C1)-(C4) are satisfied for this system by direct computation.

\section{The Mathematical Model}

We introduce the simplest model of a power system. The equations resemble a system of differential equations describing a set of coupled pendulums with constant forcing. Recently [9], [10], [16], [17], [32] this model has been subjected to serious theoretical analysis to assist in understanding its dynamical behavior.

The model consists of three main components: generators, a transmission network and loads (Fig. 1). We assume that the transmission network has $(n+m)$ nodes numbered $1, \cdots, n, n+1, \cdots, n+m$ with 0 as a reference (datum). A generator is connected to each node 1 through $n$, while an impedance load is connected to every node (Fig. 1).

We perform a standard network reduction on the network, retaining as nodes only the internal nodes of the $n$ generators. The swing equations which express the generator dynamics under the assumptions of constant rotor winding flux, constant mechanical torque, and the absence of voltage regulators are as follows (see [6], [19], [32] for details):

$$
\begin{aligned}
\frac{d}{d t} \delta_{i} & =\omega_{i}-\omega_{R} \\
M_{i} \frac{d}{d t} \omega_{i}+D_{i} \omega_{i} & =P_{m i}-P_{e i}, \quad i=1, \cdots, n
\end{aligned}
$$

where,

$M_{k}$ inertia constant,

$D_{k} \quad$ damping constant,

$P_{m k}$ constant mechanical power (torque) input,

$P_{e k} \quad$ electrical power (torque) output demanded by the network,

$\delta_{k} \quad$ the angle of the internal complex voltage or the torque angle of the $k$ th machine,

$\omega_{k}$ the rotor angular velocity of the $k$ th machine,

$\omega_{R}$ the reference frequency of the power system (usually $\omega_{R}=2 \pi 60 \mathrm{rad} / \mathrm{s}$ ).

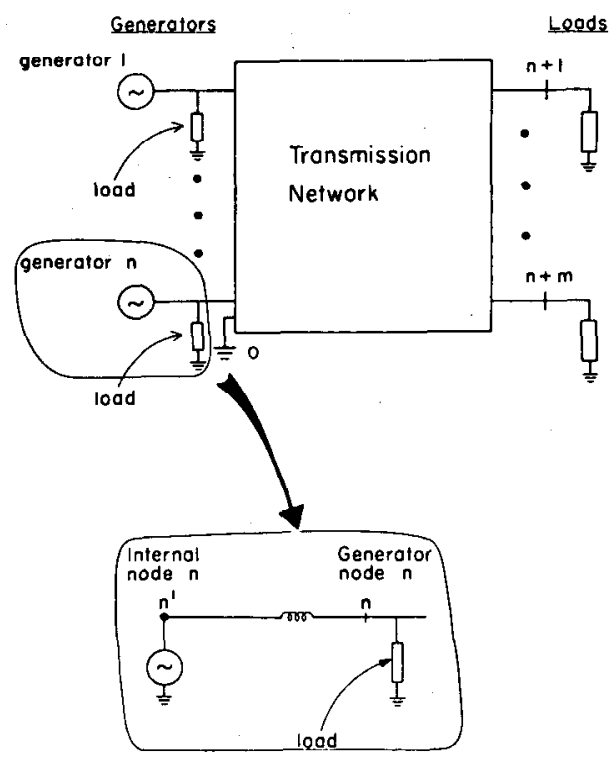

Fig. 1. A sample power system; generators, transmission network, and
loads.

The electrical power output is a function of the angle differences as follows:

$$
\begin{array}{r}
P_{e i}:=G_{i i} E_{i}^{2}+\sum_{\substack{j=1 \\
j \neq i}}^{n} E_{i} E_{j} Y_{i j} \cos \left(\Theta_{i j}-\delta_{i}+\delta_{j}\right), \\
i=1, \cdots, n
\end{array}
$$

where $G_{i i}, Y_{i j}, \Theta_{i j}$, and $E_{i}$, are all constants, defined as follows:

$E_{i}$ the magnitude of internal complex voltage (the magnitude of voltage behind the transient reactance),

$Y_{i j}$ the transfer admittance magnitude between internal nodes $i$ and $j$,

$\Theta_{i j}$ the transfer admittance phase between internal nodes $i$ and $j$,

$G_{i j}$ the total admittance at the internal node of generator $i$.

Assume $\Theta_{i j}=\pi / 2$ and assume also that the damping constants $D_{i}=0$.

If we define $P_{i}\left(:=P_{m i}-G_{i i} E_{i}^{2}\right)$ to be the exogenous specified mechanical input power and denote the constant quantity $E_{i} E_{j} Y_{i j}=: y_{i j}$, then we may write the swing equations for machine $i$ as

$$
\begin{aligned}
\dot{\delta}_{i} & =\omega_{i}-\omega_{R} \\
M_{i} \dot{\omega}_{i} & =P_{i}-\sum_{\substack{j=1 \\
j \neq i}}^{n} y_{i j} \sin \left(\delta_{i}-\delta_{j}\right) .
\end{aligned}
$$

We note that the system of equations (3.1), (3.2) describe an $n$-degree-of-freedom Hamiltonian system with the energy function:

$$
\boldsymbol{W}=\sum_{i=1}^{n} \frac{1}{2} M_{i}\left(\omega_{i}-\omega_{R}\right)^{2}-\sum_{i=1}^{n} P_{i} \delta_{i}-\sum_{i<j} y_{i j} \cos \left(\delta_{i}-\delta_{j}\right) .
$$

This energy function is Hamiltonian on the covering space 
$R^{2 n}$, but only locally Hamiltonian on the "true" space $R^{n} \times T^{n}$ where the $\delta_{i}$ are considered modulo $2 \pi$.

The energy function $W$ has been utilized as a Lyapunov function to determine an estimate of the region of attraction of a stable equilibrium point of the swing equations (see Willems [41], El-Abiad and Nagapan [18], Fouad [19], Pai [32]).

Assume that the mechanical power produced is totally absorbed by the network, i.e.,

$$
\sum_{i=1}^{n} P_{i}=0
$$

Summing up (3.2.i) gives

$$
\sum_{i=1}^{n} M_{i} \dot{\omega}_{i}=\sum_{i=1}^{n} P_{i}=0
$$

Integrating with respect to time gives

$$
\sum_{i=1}^{n} M_{i} \omega_{i}(t)=C_{1} \quad \text { (constant) }
$$

which when evaluated at the initial time $t=0$ equals

$$
\sum_{i=1}^{n} M_{i} \omega_{i}(0)=C_{1} \text {. }
$$

Using (3.1) and the constraint (3.4), one obtains

$$
\sum_{i=1}^{n} M_{i} \dot{\delta}_{i}(t)=C_{1}-\omega_{R} \sum_{i=1}^{n} M_{i}
$$

Let us define the total inertia of the system to be the sum of the individual inertias, i.e.,

$$
M:=\sum_{i=1}^{n} M_{i} .
$$

Integrating the constraint equation (3.5) with respect to time, we obtain

$$
\sum_{i=1}^{n} M_{i} \delta_{i}(t)=C_{1} t-\left(\omega_{R} M\right) t+C_{2}
$$

Rewriting the constraints (3.4) and (3.6) and replacing the constants by their values one gets

$$
\begin{aligned}
& \sum_{i=1}^{n} M_{i} \omega_{i}(t)=\sum_{i=1}^{n} M_{i} \omega_{i}(0) \\
& \sum_{i=1}^{n} M_{i} \delta_{i}(t)=\sum_{i=1}^{n} M_{i} \delta_{i}(0)+t\left(\sum_{i=1}^{n} M_{i} \omega_{i}(0)-M \omega_{R}\right)
\end{aligned}
$$

Equations (3.7) and (3.8) are time-dependent constraints. We make a coordinate change to transform these constraints to time-independent constraints, thus simplifying the system of equations. These constraint equations reduce the space of the dynamical motion by one degree of freedom or two dimensions, as is seen below.

Define the following transformation:

$$
\begin{aligned}
\omega_{i} & \rightarrow \bar{\omega}_{i}=\omega_{i}-\frac{1}{M} \sum_{j=1}^{n} M_{j} \omega_{j}(0) \\
\delta_{i} & \rightarrow \bar{\delta}_{i}=\delta_{i}+t\left(\omega_{R}-\frac{1}{M} \sum_{j=1}^{n} M_{j} \omega_{j}(0)\right)-\frac{1}{M} \sum_{j=1}^{n} M_{j} \delta_{j}(0)
\end{aligned}
$$

where we have employed the so called center-of-angle reference frame without transforming to center-of-angle coordinates. Our transformation is analogous to the one utilized by Kopell and Washburn [29] except for the factor of $(1 / M)$.

With this transformation and dropping the overbars we can summarize the autonomous swing equations with constraints as follows:

$$
\begin{aligned}
\dot{\delta}_{i} & =\omega_{i} \\
M_{i} \dot{\omega}_{i} & =P_{i}-\sum_{\substack{j=1 \\
j \neq i}}^{n} y_{i j} \sin \left(\delta_{i}-\delta_{j}\right), \quad 1 \leqslant i \leqslant n
\end{aligned}
$$

with the time-invariant constraints

$$
\begin{aligned}
\sum_{i=1}^{n} M_{i} \delta_{i} & =0 \\
\sum_{i=1}^{n} M_{i} \omega_{i} & =0
\end{aligned}
$$

and the energy (Hamiltonian) function

$$
W=\sum_{i=1}^{n} \frac{1}{2} M_{i} \omega_{i}^{2}-\sum_{i=1}^{n} P_{i} \delta_{i}-\sum_{i<j} \sum_{i j} y_{i j} \cos \left(\delta_{i}-\delta_{j}\right) .
$$

From the constraint equations (3.11) and (3.12) it is observed that the system can be reduced by one degree of freedom. This procedure of eliminating a degree of freedom by making use of a conservation law is a special case of the procedure of reduction; see Abraham and Marsden $[4$, ch. 4].

\section{Perturbation and Scaling Parameters}

We begin by choosing "transfer" parameters for the swing equations model.

\subsection{Transfer Susceptance Parameters}

Choose the coupling parameters $y_{i j}, i, j=1,2, \cdots, n-1$ to be very small (i.e., weak coupling) of order $\epsilon, \epsilon>0$, (how small $\epsilon$ must be, is determined later). Let (recall $y_{i j}=y_{j i}$ )

$$
y_{i j}=\epsilon B_{i j}, \quad 1 \leqslant i, j \leqslant n-1 \quad i \neq j
$$

and

$$
y_{i n}=B_{i n}, \quad 1 \leqslant i \leqslant n-1 .
$$

Then our system of equations becomes

$$
\begin{aligned}
\dot{\delta}_{k} & =\omega_{k}, \quad 1 \leqslant k \leqslant n \\
M_{i} \dot{\omega}_{i} & =P_{i}-\epsilon \sum_{\substack{j=1 \\
j \neq i}}^{n-1} B_{i j} \sin \left(\delta_{i}-\delta_{j}\right)-B_{i n} \sin \left(\delta_{i}-\delta_{n}\right) \\
M_{n} \dot{\omega}_{n} & =P_{n}-\sum_{j=1}^{n-1} B_{n j} \sin \left(\delta_{n}-\delta_{j}\right)
\end{aligned}
$$


with time-invariant constraints

$$
\begin{array}{lll}
\sum_{i=1}^{n} M_{i} \delta_{i}=0 & \text { or } & \delta_{n}=-\sum_{j=1}^{n-1} \frac{M_{j}}{M_{n}} \delta_{j} \\
\sum_{i=1}^{n} M_{i} \omega_{i}=0 & \text { or } & \omega_{n}=-\sum_{j=1}^{n-1} \frac{M_{j}}{M_{n}} \omega_{j}
\end{array}
$$

and the Hamiltonian

$$
\begin{aligned}
W^{\epsilon}\left(\delta_{1}, \cdots, \delta_{n}, \omega_{1}, \cdots, \omega_{n}\right) & \\
= & \sum_{j=1}^{n-1} \frac{1}{2} M_{j} \omega_{j}^{2}+\frac{1}{2} M_{n} \omega_{n}^{2}-\sum_{j=1}^{n-1} P_{j} \delta_{j}-P_{n} \delta_{n} \\
& -\epsilon \sum_{i<j}^{n-1} B_{i j} \cos \left(\delta_{i}-\delta_{j}\right)-\sum_{j=1}^{n-1} B_{j n} \cos \left(\delta_{j}-\delta_{n}\right) .
\end{aligned}
$$

\subsection{The Case of Uncoupled Machines}

Consider the case $\epsilon=0$. This corresponds to machines $1, \cdots, n-1$ being connected to machine $n$ but not to each other. From (4.3), (4.4), and (4.5),

$$
\begin{aligned}
& W^{0}\left(\delta_{1}, \cdots, \delta_{n-1}, \omega_{1}, \cdots, \omega_{n-1}\right) \\
& =\sum_{j=1}^{n-1} \frac{1}{2} M_{j} \omega_{j}^{2}+\frac{1}{2} M_{n}\left(-\sum_{k=1}^{n-1} \frac{M_{k}}{M_{n}} \omega_{k}\right)^{2}-\sum_{j=1}^{n-1} P_{j} \delta_{j} \\
& \quad+P_{n}\left(\sum_{k=1}^{n-1} \frac{M_{k}}{M_{n}} \delta_{k}\right)-\sum_{j=1}^{n-1} B_{j n} \cos \left(\delta_{j}+\sum_{k=1}^{n-1} \frac{M_{k}}{M_{n}} \delta_{k}\right) .
\end{aligned}
$$

Our next main concern is to evaluate the effect of the two states $\delta_{n}$ and $\omega_{n}$ on the rest of the system. These states are functions of the rest of the states subscripted $1, \cdots, n-1$ via (4.3) and (4.4) and thus they produce the coupling of (4.6).

$\omega_{n}$ and $\delta_{n}$ shall be restricted to be periodic with small amplitude. This implies that the coupling Hamiltonian of (4.6) shall be of the same small order and hence one obtains the Hamiltonian formulation of Arnold diffusion as in Section II (see also [26] and [1]). In the power systems context this amounts to considering the effect of the largest (infinite) machine on the system.

\subsection{Determination of the Critical Set}

We now locate the critical points of the energy function $W^{0}$. They must satisfy the following equations:

$$
\begin{aligned}
\frac{\partial W^{0}}{\partial\left(M_{j} \omega_{j}\right)}= & \frac{1}{M_{j}}\left[M_{j} \omega_{j}+M_{n} \omega_{n} \frac{d \omega_{n}}{d \omega_{j}}\right]=0 \\
\frac{\partial W^{0}}{\partial \delta_{j}}= & -P_{j}+B_{j n} \sin \left(\delta_{j}-\delta_{n}\right)+\left(\frac{d \delta_{n}}{d \delta_{j}}\right) \\
& \cdot\left[-P_{n}-\sum_{i=1}^{n-1} B_{\mathrm{in}} \sin \left(\delta_{1}-\delta_{n}\right)\right]=0 .
\end{aligned}
$$

Recall from Section II that $P_{n}=-\sum_{j}^{n-1} P_{j}$, so we may write the critical set equations as follows:

$$
\begin{aligned}
\frac{\partial W^{0}}{\partial\left(M_{j} \omega_{j}\right)}= & \omega_{j .}+\sum_{k=0}^{n-1} \frac{M_{k}}{M_{n}} \omega_{k}=0 \\
\frac{\partial W^{0}}{\partial \delta_{j}}= & -P_{j}+B_{j n} \sin \left(\delta_{j}+\sum_{k=0}^{n-1} \frac{M_{k}}{M_{n}} \delta_{k}\right) \\
& -\frac{M_{j}}{M_{n}}\left[\sum_{i=1}^{n-1}\left(P_{i}-B_{\text {in }} \sin \left(\delta_{i}-\delta_{n}\right)\right]=0\right.
\end{aligned}
$$

or in a matrix form

$$
\begin{aligned}
{\left[\begin{array}{c}
\frac{\partial W^{0}}{\partial\left(M_{j} \omega_{j}\right)} \\
\frac{\partial W^{0}}{\partial \delta_{j}}
\end{array}\right]=\left[\begin{array}{ll}
A & 0 \\
0 & B
\end{array}\right] } \\
\\
\cdot\left[\begin{array}{c}
\omega_{j} \\
\left.-P_{j}+B_{j n} \sin \left(\delta_{j}+\sum_{k=1}^{n=1} \frac{M_{k}}{M_{n}} \delta_{k}\right)\right]=0
\end{array}\right.
\end{aligned}
$$

with the elements of $A$ given by

$a_{j j}=1+\frac{M_{j}}{M_{n}} \quad$ and $\quad a_{j k}=\frac{M_{k}}{M_{n}}, \quad$ for all $j, k$ and $j \neq k$.

Similarly, the elements of $B$ are

$b_{j j}=1+\frac{M_{j}}{M_{n}} \quad$ and $\quad b_{j k}=\frac{M_{j}}{M_{n}}, \quad$ for all $j, k$ and $j \neq k$.

Therefore, the $2(n-1) \times 2(n-1)$ matrix

$$
\left[\begin{array}{cc}
A & 0 \\
0 & B
\end{array}\right]
$$

is nonsingular with determinant $>1$. Thus equations (4.7) and (4.8) are equivalent to the following simpler conditions:

$$
\begin{aligned}
\omega_{i} & =0 \\
P_{i}-B_{i n} \sin \left(\delta_{i}+\sum_{k=1}^{n-1} \frac{M_{k}}{M_{n}} \delta_{k}\right) & =0, \quad 1 \leqslant i \leqslant n-1 .
\end{aligned}
$$

Let

$$
\begin{gathered}
\Delta_{i}:=\delta_{i}+\sum_{k=1}^{n-1} \frac{M_{k}}{M_{n}} \delta_{k}, \\
\Delta_{i}^{s}:=\delta_{i}^{s}+\sum_{k=1}^{n-1} \frac{M_{k}}{M_{n}} \delta_{k}^{s}
\end{gathered}
$$

to be the mod- $(2 \pi)$ constant "angle" such that $(4.10 . \mathrm{i})$ is satisfied for all $i=1, \cdots, n-1$. Assume that the point $\left(\left(\Delta_{l}^{s}, 0\right), i=1, \cdots, n-1\right)$ is a local minimum of the energy function $W^{0}$ (or a stable point of the differential equation, see $[8,16,37]$ for a detailed treatment of the critical set). One can verify that every point of the critical set is non-degenerate (the Hessian matrix of $W^{0}$ is nonsingular).

We remark that the $\Delta_{i}^{s}, i=1, \cdots, n-1$, uniquely determine the $\delta_{k}^{s}, k=1, \cdots, n-1$, i.e., the matrix defining 


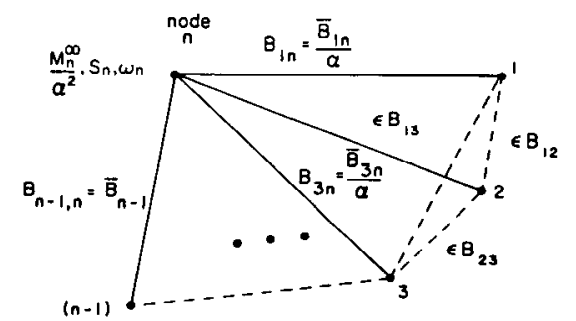

Fig. 2. The power system diagram (our model).

the linear map $\left(\delta_{1}^{s}, \cdots, \delta_{n-1}^{s}\right) \rightarrow\left(\Delta_{1}^{s}, \cdots, \Delta_{n-1}^{s}\right)$ of $(4.11)$ is invertible.

Let us now define as new state variables $(\sigma, \omega)$ where

$\boldsymbol{\sigma}=\left(\sigma_{1}, \cdots, \sigma_{n-1}\right), \quad$ with $\quad \sigma_{k}=\delta_{k}-\delta_{k}^{s} \quad 1 \leqslant k \leqslant n-1$, and $\omega=\left(\omega_{1}, \cdots, \omega_{n-1}\right)$

is the same as before. Then we rewrite the Hamiltonian as follows:

$$
\begin{aligned}
\boldsymbol{H}(\boldsymbol{\sigma}, \omega)= & \sum_{i=1}^{n-1} \frac{1}{2} M_{i} \omega_{i}^{2}+\frac{1}{2} M_{n}\left[-\sum_{k=1}^{n-1} \frac{M_{k}}{M_{n}} \omega_{k}\right]^{2} \\
& -\sum_{i=1}^{n-1} P_{i} \sigma_{i}+P_{n}\left[\sum_{k=1}^{n-1} \frac{M_{k}}{M_{n}} \sigma_{k}\right] \\
& -\sum_{i=1}^{n-1} B_{i n}\left[\cos \left(\sigma_{i}+\sum_{k=1}^{n-1} \frac{M_{k}}{M_{n}} \sigma_{k}+\Delta_{i}^{s}\right)-\cos \left(\Delta_{i}^{s}\right)\right]
\end{aligned}
$$

with $\boldsymbol{H}(0,0)=0$.

\subsection{The Choice of Constants (Parameters)}

Suppose $M_{n-1} \ll M_{1}, \cdots$, and $M_{n-2} \ll M_{n}$. Let us express this condition by introducing a nonzero small parameter $\alpha$ such that

$$
M_{n}:=\frac{M_{n}^{\infty}}{\alpha^{2}}, \quad M_{n-1}:=\bar{M}_{n-1}, \quad \text { and } \quad M_{i}:=\frac{\bar{M}_{i}}{\alpha},
$$

$1 \leqslant i \leqslant n-2$

where the overbarred quantities are of the same order $(0(1))$ and $M_{n}^{\infty}$ is large. Denote

$$
\begin{aligned}
\mu_{i} & =\frac{\bar{M}_{i}}{M_{n}^{\infty}}, \quad 1 \leqslant i \leqslant n-2 \\
\mu_{n-1} & =\alpha \bar{\mu}_{n-1}=\alpha \frac{\bar{M}_{n-1}}{M_{n}^{\infty}} .
\end{aligned}
$$

Note that for an infinite machine (i.e., $M_{n}$ or $M_{n}^{\infty} \rightarrow \infty$ ), we have $\mu_{i} \rightarrow 0,1 \leqslant i \leqslant n-1$. We further require that each "large" machine is connected to the $n$th machine via a "strong" line (see Fig. 2), i.e., we let

$$
B_{i n}=\frac{\bar{B}_{i}}{\alpha}, \quad 1 \leqslant i \leqslant n-2 \text { and } B_{n-1, n}=\bar{B}_{n-1} .
$$

Correspondingly, let

$$
P_{n}=\frac{\bar{P}_{n}}{\alpha}, \quad P_{i}=\frac{\bar{P}_{i}}{\alpha}, \quad 1 \leqslant i \leqslant n-2 \quad \text { and } \quad P_{n-1}=\bar{P}_{n-1} \text {. }
$$

Let $\mu=\left(\mu_{1}, \cdots, \mu_{n-2}, \bar{\mu}_{n-1}\right)$, and rewrite the Hamiltonian function as

$$
\begin{aligned}
& H^{(\mu, \alpha)} \\
& =\sum_{i=1}^{n-2} \frac{1}{2} \frac{\bar{M}_{i}}{\alpha} \omega_{i}^{2}+\frac{1}{2} \bar{M}_{n-1} \omega_{n-1}^{2}+\frac{1}{2} \frac{M_{n}^{\infty}}{\alpha^{2}}\left[\alpha \sum_{k-1}^{n-1} \mu_{k} \omega_{k}\right]^{2} \\
& \quad-\sum_{i=1}^{n-2} \frac{\bar{P}_{i}}{\alpha} \sigma_{i}-\bar{P}_{n-1} \sigma_{n-1}+\frac{\bar{P}_{n}}{\alpha}\left[\sum_{k=1}^{n-1} \alpha \mu_{k} \sigma_{k}\right] \\
& -\sum_{i=1}^{n-2} \frac{\bar{B}_{i}}{\alpha}\left[\cos \left(\sigma_{i}+\alpha \sum_{k=1}^{n-1} \mu_{k} \sigma_{k}+\Delta_{i}^{s}\right)-\cos \left(\Delta_{i}^{s}\right)\right] \\
& -\bar{B}_{n-1}\left[\cos \left(\sigma_{n-1}+\alpha \sum_{k=1}^{n-1} \mu_{k} \sigma_{k}+\Delta_{n-1}^{s}\right)-\cos \left(\Delta_{n-1}^{s}\right)\right] .
\end{aligned}
$$

One may observe from (4.12) that the coupling between subsystems $1, \cdots, n-1$ is due to the states $\delta_{n}$ and $\omega_{n}$ of the $n$th machine. That is, if $M_{n}^{\infty}=\infty$ then the parameters $\mu_{k}=0, k=1, \cdots, n-1$ and one obtains the decoupled ('unperturbed') Hamiltonian. For notational simplicity let $\mu_{1}=\cdots=\mu_{n-2}=\bar{\mu}_{n-1}=\mu$.

The unperturbed Hamiltonian $(\mu=0)$ is

$$
\begin{aligned}
H^{(0, \alpha)}= & \sum_{i=1}^{n-2} \frac{1}{2} \frac{\bar{M}_{i}}{\alpha} \omega_{i}^{2}-\sum_{i=1}^{n-2} \frac{\bar{P}_{i}}{\alpha} \sigma_{i} \\
& -\sum_{i=1}^{n-2} \frac{\bar{B}_{i}}{\alpha}\left[\cos \left(\sigma_{i}+\Delta_{i}^{s}\right)-\cos \left(\Delta_{i}^{s}\right)\right] \\
& +\left\{\frac{1}{2} \bar{M}_{n-1} \omega_{n-1}^{2}-\bar{P}_{n-1} \sigma_{n-1}\right. \\
& \left.-\bar{B}_{n-1}\left[\cos \left(\sigma_{n-1}+\Delta_{n-1}^{s}\right)-\cos \left(\Delta_{n-1}^{s}\right)\right]\right\} .
\end{aligned}
$$

We note that the perturbation affects the critical set: the unperturbed system has the point $\left(\Delta_{1}^{s}, \cdots, \Delta_{n-1}^{s}, \mathbf{0}\right)$ as a critical point. But after perturbation this critical point is transformed to the point $\left(\delta_{1}^{s}, \cdots, \delta_{n-1}^{s}, \mathbf{0}\right)$ via (4.11). Thus it is necessary to use the Melnikov version developed by Holmes and Marsden [26] rather than that of Arnold [11].

The perturbation parameter is $\mu$ and the purpose of the parameter $\alpha$ is explained as follows. The unperturbed (i.e., $\mu=0$ ) Hamiltonian system describes a system of pendulums with constant forcing. The phase portrait of each of its subsystems is thoroughly discussed in Andronov and Chaikin [7, p. 293]. Under the assumptions that $\bar{P}_{j}<\bar{B}_{j}$, $1 \leqslant j \leqslant n-1$, one obtains the phase portrait for each subsystem $j$ as in Fig. 3.

The parameter $\alpha$ is selected to boost the energy values of the level curves of subsystem $i, 1 \leqslant i \leqslant n-2$, compared to the energy values of the level curves of the subsystem $n-1$. More precisely let the energy constant of the separatrix (or homoclinic orbit) of subsystem $n-1$ be $\bar{h}$. Then one chooses $\alpha$ small enough such that the total (system's) unperturbed Hamiltonian, with an energy constant $h>\bar{h}$ and $h$ close to $\bar{h}$, possesses solution curves which are cross products of one homoclinic orbit (that of subsystem $n-1$ ) 


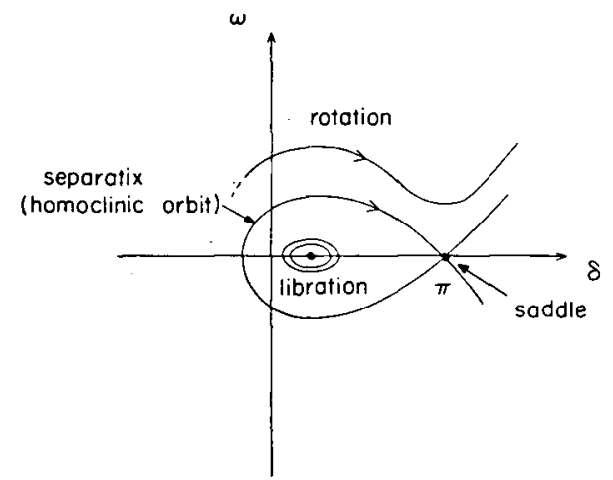

Fig. 3. The phase portrait of a pendulum with constant forcing.

and $(n-2)$ closed orbits (those of subsystem $i, 1 \leqslant i \leqslant n-$ 2).

As a consequence of this choice of parameters, the Hamiltonian system will satisfy the first three conditions of Section II (i.e., (C1)-(C3)). Also note that the combined subsystems $i, 1 \leqslant i \leqslant n-2$ admit, implicitly, action-angle coordinates (see Arnold [12, p. 285]).

We now scale the perturbed Hamiltonian $H^{(\mu, \alpha)}$, (equation 4.12$)$, by multiplying through by $\left(\alpha / \bar{M}_{j}\right)$, where $\bar{M}_{j}$ : $1 \leqslant j \leqslant n-1$ are of the same order $(0(1)$ ) (for clarity, assume all $\bar{M}_{j}$ are the same). We expand in powers of the small perturbation parameter $\mu$ to obtain the following (expanded) Hamiltonian:

$$
\begin{aligned}
\boldsymbol{H}^{\mu}= & \sum_{i=1}^{n-2} \frac{1}{2} \omega_{i}^{2}-\rho_{i} \sigma_{i}-\beta_{i}\left[\cos \left(\sigma_{i}+\Delta_{i}^{s}\right)-\cos \left(\Delta_{i}^{s}\right)\right] \\
& +\alpha\left[\frac{1}{2} \omega_{n-1}^{2}-\rho_{n-1} \sigma_{n-1}\right. \\
& \left.-\beta_{n-1}\left[\cos \left(\sigma_{n-1}+\Delta_{n-1}^{s}\right)-\cos \left(\Delta_{n-1}^{s}\right)\right]\right] \\
& +\alpha \mu \frac{1}{2}\left[\sum_{i=1}^{n-2} \omega_{i}+\alpha \omega_{n-1}\right]^{2}+\alpha \mu\left[\sum_{i=1}^{n-2} \rho_{n} \sigma_{i}+\alpha \rho_{n} \sigma_{n-1}\right] \\
& +\alpha \mu \sum_{i=1}^{n-2} \beta_{i} \sin \left(\sigma_{i}+\Delta_{i}^{s}\right)\left[\sum_{k=1}^{n-2} \sigma_{k}+\alpha \sigma_{n-1}\right]+0\left(\alpha^{2} \mu^{2}\right) \\
& +\alpha^{2} \mu \beta_{n-1} \sin \left(\sigma_{n-1}+\Delta_{n-1}^{s}\right)\left[\sum_{k=1}^{n-2} \sigma_{k}+\alpha \sigma_{n-1}\right] \\
& +0\left(\alpha^{3} \mu^{2}\right)
\end{aligned}
$$

where $\rho_{k}=\bar{P}_{k} / \bar{M}_{k}, \beta_{k}=\bar{B}_{k} / \bar{M}_{k}$, and so on.

At this point one may identify the subsystems as follows:

(A) The subsystem $(n-1)$,

$$
\begin{aligned}
\alpha \boldsymbol{F}\left(\sigma_{n-1}, \omega_{n-1}\right)= & \alpha\left(\frac{1}{2} \omega_{n-1}^{2}-\rho_{n-1} \sigma_{n-1}\right. \\
& \left.-\beta_{n-1}\left[\cos \left(\sigma_{n-1}+\Delta_{n-1}^{s}\right)-\cos \left(\Delta_{n-1}^{s}\right)\right]\right)
\end{aligned}
$$

(the Hamiltonian of a pendulum with constant forcing), which possesses the homoclinic orbit denoted $\left(\bar{\sigma}_{n-1}, \bar{\omega}_{n-1}\right)$ and shown in Fig. 4. Let $F\left(\bar{\sigma}_{n-1}, \bar{\omega}_{n-1}\right)=\bar{h}$.

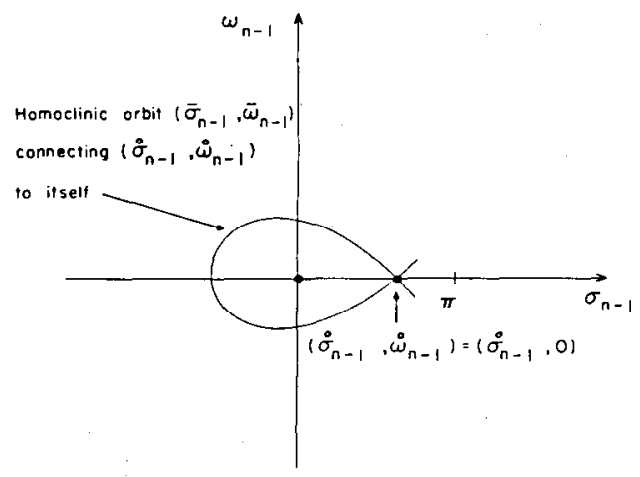

Fig. 4. A homoclinic orbit (subsystem $n-1$ ).

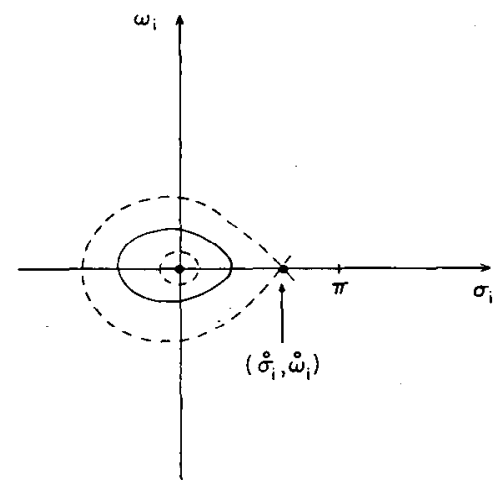

Fig. 5. Closed orbits of a pendulum (subsystem $i, 1 \leqslant i \leqslant n-2$ ).

(B) For each $i, i=1, \cdots, n-2$, the subsystem (i)

$$
G_{i}\left(\sigma_{i}, \omega_{i}\right)=\frac{1}{2} \omega_{i}^{2}-\rho_{i} \sigma_{i}-\beta_{i}\left[\cos \left(\sigma_{i}+\Delta_{i}^{s}\right)-\cos \left(\Delta_{i}^{s}\right)\right]
$$

which is a nonlinear oscillator with amplitude-dependent frequencies (this is a property of the closed orbits of a pendulum phase portrait (see Fig. 5)). Denote a solution curve of the oscillator $i$, which is a projection of a homoclinic orbit of the total unperturbed system on the subspace $i$, by $\left(\bar{\sigma}_{i}, \bar{\omega}_{i}\right)$.

(AB) Finally we identify the terms of order $\mu$ as a perturbation energy function, $\boldsymbol{H}^{1}$,

$$
\begin{aligned}
\boldsymbol{H}^{1}= & \frac{1}{2} \alpha\left[\sum_{i=1}^{n-2} \omega_{i}+\alpha \omega_{n-1}\right]^{2}+\alpha\left[\sum_{i=1}^{n-2} \rho_{n} \sigma_{i}+\alpha \rho_{n} \sigma_{n-1}\right] \\
& +\alpha \sum_{i=1}^{n-2} \beta_{i} \sin \left(\sigma_{i}+\Delta_{i}^{s}\right)\left[\sum_{k=1}^{n-2} \sigma_{k}+\alpha \sigma_{n-1}\right] \\
& +\alpha^{2} \beta_{n-1} \sin \left(\sigma_{n-1}+\Delta_{n-1}^{s}\right)\left[\sum_{k=1}^{n-2} \sigma_{k}+\alpha \sigma_{n-1}\right] .
\end{aligned}
$$

\section{The Existence of ARNOLd Diffusion}

We now investigate whether the conditions of Section II can be verified. First one notes that the solutions of each subsystem $i, 1 \leqslant i \leqslant n-2$, are all closed orbits. Thus the solutions of the combined $(n-2)$-subsystems lie on $(n-2)$ dimensional tori and a transformation to action-angle vari- 
ables is possible. In fact for each subsystem $i$ the action is

$$
I_{i}\left(\bar{h}_{i}\right)=\frac{1}{\pi} \int_{\sigma_{i_{\min }}}^{\sigma_{i_{\max }}}\left[2\left(\bar{h}_{i}+\rho_{i} \sigma_{i}+\beta_{i} \cos \left(\sigma_{i}+\Delta_{i}^{s}\right)\right]^{1 / 2} d \sigma_{i}\right.
$$

where

$$
\bar{h}_{i}:=h_{i}-\cos \left(\Delta_{i}^{s}\right), \quad \bar{h}_{i}<-\rho_{i}\left(\pi-\Delta_{i}^{s}\right)+\beta_{i} \cos \left(\Delta_{i}^{s}\right)
$$$$
\begin{array}{cl}
h_{i} & \text { energy constant such that } G_{i}\left(\sigma_{i}, \omega_{i}\right)=h_{i} \\
\sigma_{i_{\max }}\left(\sigma_{i_{\min }}\right) & \text { is the maximum (minimum) value of } \sigma_{i} \\
& \text { at which the energy level curve, } G_{i}\left(\sigma_{i}, \omega_{i}\right) \\
& =h_{i} \text { crosses the } \sigma_{i} \text {-axis. }
\end{array}
$$

If $\rho_{i}=0$, the integral (5.1.i) may be transformed into an elliptic integral which can be looked up in Integral Tables.

Secondly, the frequencies $\Omega_{i}$ of the closed orbits of each subsystem $i, 1 \leqslant i \leqslant n-2$, are amplitude dependent. Therefore, one may select energy constants $G_{i}\left(\sigma_{i}, \omega_{i}\right)=h_{i}, 1 \leqslant i$ $\leqslant n-2$, such that these frequencics arc rationally independent. Thus the first three conditions of Section II ((C1), (C2), and (C3)) are clearly satisfied. Now we seek to satisfy condition (C4), so we compute the Melnikov integrals.

Using the perturbed Hamiltonian $\boldsymbol{H}^{\mu},(4.14)$, and evaluating the integrals along a homoclinic orbit $\left(\bar{\sigma}_{1}, \cdots, \bar{\sigma}_{n-1}, \bar{\omega}_{1}, \cdots, \bar{\omega}_{n-1}\right)$, one obtains

$$
\begin{aligned}
\tilde{M}_{i}:= & \int_{-\infty}^{\infty}\left\{G_{i}, H^{1}\right\} d t \\
= & \int_{-\infty}^{\infty}-\bar{\omega}_{i}\left(\alpha\left(-\beta_{i} \cos \left(\bar{\sigma}_{i}+\Delta_{i}^{s}\right)\left[\sum_{k=1}^{n-2} \bar{\sigma}_{k}+\alpha \bar{\sigma}_{n-1}\right]\right)\right. \\
& +\alpha\left(\sum_{j=1}^{n-2} \rho_{j}-\beta_{j} \sin \left(\bar{\sigma}_{j}+\Delta_{j}^{s}\right)\right) \\
& \left.+\alpha^{2}\left(\rho_{n-1}-\beta_{n-1} \sin \left(\bar{\sigma}_{n-1}+\Delta_{n-1}^{s}\right)\right)\right) \\
& +\alpha\left[\sum_{k=1}^{n-2} \bar{\omega}_{k}+\alpha \bar{\omega}_{n-1}\right]\left(\rho_{i}-\beta_{i} \sin \left(\bar{\sigma}_{i}+\Delta_{i}^{s}\right)\right) d t .
\end{aligned}
$$

We note that only a product of the oscillators variables (subscripted $i, 1 \leqslant i \leqslant n-2$ ) with the homoclinic orbit variables (subscripted $(n-1)$ ) would produce nonvanishing terms.

The Melnikov integral measures the separation between the stable and unstable manifolds by measuring the energy differences along two curves which are, respectively, asymptotic to the invariant torus as $t \rightarrow \infty$ and $t \rightarrow-\infty$. Because these curves need not be close on the torus, the limits of integration must be chosen carefully. Such terms arise from products of oscillator variables in (5.2.i). These terms are zero when the appropriate limits are chosen, and so may be omitted from (5.2.i) - see Appendix A. (A similar phenomena occurs in the pendulum oscillator example of Holmes and Marsden [26] although they did not discuss it). Hence the integral of (5.2.i) above reduces to

$$
\begin{aligned}
\tilde{\boldsymbol{M}}_{i}= & \int_{-\infty}^{\infty}\left\{-\alpha^{2}\left(\frac{d}{d t}\left[\rho_{i}-\beta_{i} \sin \left(\bar{\sigma}_{i}+\Delta_{i}^{s}\right)\right]\right) \bar{\sigma}_{n-1}\right. \\
& +\alpha^{2}\left[\rho_{i}-\beta_{i} \sin \left(\bar{\sigma}_{i}+\Delta_{i}^{s}\right)\right] \bar{\omega}_{n-1} \\
& \left.-\alpha^{2}\left[\rho_{n-1}-\beta_{n-1} \sin \left(\bar{\sigma}_{n-1}+\Delta_{n-1}^{s}\right)\right] \bar{\omega}_{i}\right\} d t .
\end{aligned}
$$

One may divide by $\alpha^{2}$ to obtain the Melnikov integral independent of the (scaling) parameter $\alpha$. Letting

$$
\begin{aligned}
\hat{M}_{i}= & \frac{\tilde{M}_{i}}{\alpha^{2}} \\
\hat{M}_{i}= & \int_{-\infty}^{\infty}\left\{-\left(\frac{d}{d t}\left[\rho_{i}-\beta_{i} \sin \left(\bar{\sigma}_{i}\left(\Omega_{i}\left(t-t_{i}\right)\right)+\Delta_{i}^{s}\right)\right]\right) \bar{\sigma}_{n-1}(t)\right. \\
& +\left[\rho_{i}-\beta_{i} \sin \left(\bar{\sigma}_{i}\left(\Omega_{i}\left(t-t_{i}\right)\right)+\Delta_{i}^{s}\right)\right] \bar{\omega}_{n-1}(t) \\
& -\left[\rho_{n-1}-\beta_{n-1} \sin \left(\bar{\sigma}_{n-1}(t)+\Delta_{n-1}^{s}\right)\right] \\
& \left.\cdot \bar{\omega}_{i}\left(\Omega_{i}\left(t-t_{i}\right)\right)\right\} d t
\end{aligned}
$$

where the variables $\bar{\sigma}_{i}, \bar{\omega}_{i}$ are periodic in $t$, and with (amplitude dependent) frequency $\Omega_{i}$. Note that

$$
\bar{\omega}_{i}\left(\Omega_{i} t\right)=\frac{d}{d t} \bar{\sigma}_{i}\left(\Omega_{i} t\right)
$$

Similarly, along the same homoclinic orbit $\left(\bar{\sigma}_{1}, \cdots\right.$, $\left.\bar{\sigma}_{n-1}, \bar{\omega}_{1}, \cdots, \bar{\omega}_{n-1}\right)$ one computes

$$
\begin{aligned}
\tilde{\boldsymbol{M}}_{n-1}:= & \int_{-\infty}^{\infty}\left\{\boldsymbol{F}, \boldsymbol{H}^{1}\right\} d t \\
= & \int_{-\infty}^{\infty}-\alpha \bar{\omega}_{n-1}\left\{\alpha^{2}\left(-\beta_{n-1} \cos \left(\bar{\sigma}_{n-1}+\Delta_{n-1}^{s}\right)\right)\right. \\
& \cdot\left[\sum_{k=1}^{n-2} \bar{\sigma}_{k}+\alpha \bar{\sigma}_{n-1}\right] \\
& +\alpha^{2}\left[\sum_{j=1}^{n-2} \rho_{j}-\beta_{j} \sin \left(\bar{\sigma}_{j}+\Delta_{j}^{s}\right)\right) \\
& \left.+\alpha^{3}\left(\rho_{n-1}-\beta_{n-1} \sin \left(\bar{\sigma}_{n-1}+\Delta_{n-1}^{s}\right)\right)\right\} \\
& +\alpha^{2}\left[\sum_{k=1}^{n-2} \bar{\omega}_{k}+\alpha \bar{\omega}_{n-1}\right] \\
& \cdot \alpha\left(\rho_{n-1}-\beta_{n-1} \sin \left(\bar{\sigma}_{n-1}+\Delta_{n-1}^{s}\right)\right) d t .
\end{aligned}
$$

As before, products of oscillator variables can be omitted and so only a product of variables of subscripts $\{i=$ $1, \cdots, n-2\}$ and $\{n-1\}$ contribute to the evaluation of 
the integrals. Hence the Melnikov integral reduces to

$$
\begin{aligned}
\tilde{M}_{n-1}= & \int_{-\infty}^{\infty}\left\{-\alpha^{3}\left[\frac{d}{d t}\left(\rho_{n-1}-\beta_{n-1} \sin \left(\bar{\sigma}_{n-1}+\Delta_{n-1}^{s}\right)\right)\right]\right. \\
& \cdot \sum_{k=1}^{n-2} \bar{\sigma}_{k}-\alpha^{3}\left[\sum_{j=1}^{n-2}\left(\rho_{j}-\beta_{j} \sin \left(\bar{\sigma}_{j}+\Delta_{j}^{s}\right)\right) \bar{\omega}_{n-1}\right] \\
& \left.+\alpha^{3}\left[\sum_{k=1}^{n-2}\left(\rho_{n-1}-\beta_{n-1} \sin \left(\bar{\sigma}_{n-1}+\Delta_{n-1}^{s}\right)\right) \bar{\omega}_{k}\right]\right\} d t
\end{aligned}
$$

which shows that the Melnikov function can be made independent of $\alpha$, ( $\alpha$ does not affect the transversal zeros), we, therefore, scale the Melnikov integral as follows. Let

$\hat{M}_{n-1}=\frac{\tilde{M}_{n-1}}{\alpha^{3}}$

so

$$
\begin{aligned}
\hat{M}_{n-1}= & \sum_{k=1}^{n-2} \int_{-\infty}^{\infty}\left\{-\frac{d}{d t}\left[\rho_{n-1}-\beta_{n-1} \sin \left(\bar{\sigma}_{n-1}+\Delta_{n-1}^{s}\right)\right]\right. \\
& \cdot \sigma_{k}\left(\Omega_{k}\left(t-t_{k}\right)\right) \\
& +\left[\rho_{n-1}-\beta_{n-1} \sin \left(\bar{\sigma}_{n-1}+\Delta_{n-1}^{s}\right)\right] \bar{\omega}_{k}\left(\Omega_{k}\left(t-t_{k}\right)\right) \\
& -\left[\rho_{k}-\beta_{k} \sin \left(\bar{\sigma}_{k}\left(\Omega_{k}\left(t-t_{k}\right)+\Delta_{k}^{s}\right)\right] \bar{\omega}_{n-1}(t)\right\} d t
\end{aligned}
$$

We may now state the following result.

\section{Theorem 5.1}

Let $h>\bar{h}$ be such that the subsystems $i, 1 \leqslant i \leqslant n-2$, possess closed orbits only. If the Melnikov integrals defined by (5.3) and (5.5) possess at least one transversal zero, then, for a sufficiently large machine $M_{n}$ (i.e., for $\mu$ sufficiently small), Arnold diffusion arises on the energy level $h$.

Following remark 4 on p. 672 of Holmes and Marsden [26] (which is due to Weinstein), it is noted that transversal intersection of the perturbed stable and unstable manifolds of the invariant tori occurs for almost all Hamiltonian vector fields. We note that our system has a specific vector field even though there is a freedom to change parameters (e.g., $\rho_{j}, \beta_{j}$, etc.). Thus we must test explicitly if the Melnikov integral equations possess transversal zeros.

Remark: One may consider the Fourier expansion of the closed curves $\bar{\sigma}_{k}, \bar{\omega}_{k}, k=1, \cdots, n-2$, up to any integer $J$, as

$$
\begin{aligned}
& \overline{\boldsymbol{\sigma}}_{k}\left(\Omega_{k}\left(t-t_{k}\right)\right) \\
& \approx \sum_{j=1}^{J} a_{j} \cos \left(j \Omega_{k}\left(t-\bar{t}_{k}\right)\right) \\
& \approx \sum_{j=1}^{J} a_{j}\left[\cos \left(j \Omega_{k} t\right) \cos \left(j \Omega_{k} \bar{t}_{k}\right)+\sin \left(j \Omega_{k} t\right) \sin \left(j \Omega_{k} \bar{t}_{k}\right)\right]
\end{aligned}
$$

and

$$
\begin{aligned}
\bar{\omega}_{k}\left(\Omega_{k}\left(t-t_{k}\right)\right) & \\
\approx & \sum_{j=1}^{J}-j \Omega_{k} a_{j} \sin \left(j \Omega_{k}\left(t-\bar{t}_{k}\right)\right) \\
\approx & \sum_{j=1}^{J}\left(-j a_{j} \Omega_{k}\right)\left[\sin \left(j \Omega_{k} t\right) \cos \left(j \Omega_{k} \bar{t}_{k}\right)\right. \\
& \left.-\sin \left(j \Omega_{k} \bar{t}_{k}\right) \cos \left(j \Omega_{k} t\right)\right] .
\end{aligned}
$$

This expansion seems useful for computational purposes when the unperturbed solutions $\bar{\sigma}_{k}, \bar{\omega}_{k}$ are not available in a closed analytic form. Of course one should obtain an upper bound on the error for this approximation to be meaningful. In the case when the amplitude of the periodic solutions are very small one can approximate these solutions by the first terms of the Fourier expansion, with some small error term. This is treated next.

\section{A Restricted Case to Small Oscillations}

If one requires that the solution orbits of each unperturbed subsystem $i, 1 \leqslant i \leqslant n-2$, is of a sufficiently small order of magnitude, then one can derive explicit conditions for a transversal zero.

Consider the original Hamiltonian equation (4.12) (before scaling in $\alpha$ ). Assume that $\alpha$ is sufficiently small and that the closed unperturbed solutions of each subsystem $i$, $1 \leqslant i \leqslant n-2$, is of order $\alpha$ in magnitude, i.e., one may substitute $\alpha \sigma_{i}$ (respectively, $\alpha \omega_{i}$ ) for $\sigma_{i}$ (respectively, $\omega_{i}$ ) for all $1 \leqslant i \leqslant n-2$. Let $\bar{\mu}_{n-1}, \mu_{k}=\mu, k=1, \cdots, n-2$. After cancellations and collections of terms we obtain

$$
\begin{aligned}
H_{\alpha}^{\mu}= & \alpha \sum_{i=1}^{n-2} \frac{1}{2} \bar{M}_{i} \omega_{i}^{2}+\alpha \sum_{i=1}^{n-2}\left(\frac{\bar{B}_{i}}{2} \cos \left(\Delta_{i}^{s}\right) \sigma_{i}^{2}\right) \\
& +\alpha^{2} \sum_{i=1}^{n-2} \bar{B}_{i} \cos \left(\Delta_{i}^{s}\right)\left(\sigma_{i} \sum_{k=1}^{n-1} \mu \sigma_{k}\right)+0\left(\alpha^{l} \mu^{s}\right) \\
& +\frac{1}{2} \bar{M}_{n-1} \omega_{n-1}^{2}-\bar{P}_{n-1}\left[\sigma_{n-1}+\alpha \sum_{k=1}^{n-1} \mu \sigma_{k}\right] \\
& -\bar{B}_{n-1}\left[\cos \left(\sigma_{n-1}+\Delta_{n-1}^{s}\right)-\cos \left(\Delta_{n-1}^{s}\right)\right. \\
& \left.-\alpha^{2}\left(\sum_{k=1}^{n-1} \mu \sigma_{k}\right) \sin \left(\sigma_{n-1}+\Delta_{n-1}^{s}\right)+0\left(\alpha^{l} \mu^{s}\right)\right] \\
& +\frac{1}{2} \alpha^{2} M_{n}^{\infty}\left[\sum_{k=1}^{n-1} \mu \omega_{k}\right]^{2}
\end{aligned}
$$

where $l=1$ and $s=2$. We may write the Hamiltonian in a 
scaled form.

$$
\begin{aligned}
H_{\alpha}^{\mu}= & \alpha \sum_{i=1}^{n-2} \frac{1}{2} \omega_{i}^{2}+\alpha \sum_{i=1}^{n-2}\left(\beta_{i n} \cos \Delta_{i}^{s}\right) \frac{\boldsymbol{\sigma}_{i}^{2}}{2}+\alpha^{2} \sum_{i=1}^{n-2} \beta_{i n} \cos \left(\Delta_{i}^{s}\right)\left(\mu \sigma_{i} \sum_{k=1}^{n-1} \sigma_{k}\right)+0\left(\alpha^{l} \mu^{s}\right)+\frac{1}{2} \omega_{n-1}^{2}-\bar{\rho}_{n-1}\left[\sigma_{n-1}+\alpha \mu \sum_{k=1}^{n-1} \sigma_{k}\right] \\
& -\beta_{n-1, n}\left[\cos \left(\sigma_{n-1}+\Delta_{n-1}^{s}\right)-\cos \left(\Delta_{n-1}^{s}\right)-\alpha^{2}\left(\mu \sum_{k=1}^{n-1} \sigma_{k}\right) \sin \left(\sigma_{n-1}+\Delta_{n-1}^{s}\right)+0\left(\alpha^{\prime} \mu^{s}\right)\right]+\frac{1}{2} \alpha^{2} \mu\left[\sum_{k=1}^{n-1} \omega_{k}\right]^{2}
\end{aligned}
$$

This formulation is particularly interesting since it resembles Arnold's first example of "Arnold diffusion" (see Arnold [11]). We shall show that the Hamiltonian, $H_{\alpha}^{\mu}$ will meet all the requirements of Section II.

One may consider $\mu$ as a perturbation parameter which couples the Hamiltonian system, and $\alpha$ as a small (nonzero) parameter which measures the "size" of the neighborhood of the stable equilibrium point of the decoupled ( $\mu=0)$ subsystem $i, 1 \leqslant i \leqslant n-2$, within which solutions are restricted.

For a fixed $\alpha>0$ set $\mu=0$ to obtain the decoupled system. We note that the nonlinear oscillators are amplitude dependent due to higher order terms in $\alpha$.

Again one may identify the subsystems as follows.

(a) The subsystem $n-1$ with Hamiltonian

$$
\begin{aligned}
F\left(\sigma_{n-1}, \omega_{n-1}\right):= & \frac{1}{2} \omega_{n-1}^{2}-\bar{\rho}_{n-1} \sigma_{n-1} \\
& -\beta_{n-1, n}\left[\cos \left(\sigma_{n-1}+\Delta_{n-1}^{s}\right)-\cos \left(\Delta_{n-1}^{s}\right)\right]
\end{aligned}
$$

possesses a homoclinic orbit $\left(\bar{\sigma}_{n-1}, \bar{\omega}_{n-1}\right)$ of energy level $F\left(\bar{\sigma}_{n-1}, \bar{\omega}_{n-1}\right)=\bar{h}$, and

(b) Each subsystem $i, i=1, \cdots, n-2$, with Hamiltonian

$$
G_{i}\left(\sigma_{i}, \omega_{i}\right)=\alpha\left(\frac{1}{2} \omega_{i}^{2}+\left(\beta_{i n} \cos \left(\Delta_{i}^{s}\right)\right) \frac{\sigma_{i}^{2}}{2}\right)+0\left(\alpha^{2}\right)
$$

is a nonlinear oscillator. Note that $0\left(\alpha^{2}\right)$, which is independent of $\mu$, is responsible for the amplitude dependent frequencies of which

$$
\left(\beta_{i n} \cos \left(\Delta_{i}^{s}\right)\right)^{1 / 2}
$$

is the first term of the Taylor expansion.

(c) The coupling Hamiltonian is the following function of $\mu$ and $\alpha$
Integrals of products of oscillators (subscripted $1 \leqslant i \leqslant n-$ 2) vanish as before, so (6.2.i) reduces to (see Appendix A).

$$
\begin{aligned}
\tilde{\tilde{\boldsymbol{M}}}_{i}=\int_{-\infty}^{\infty}\left(\left(\beta_{\mathrm{in}} \cos \left(\Delta_{i}^{s}\right)\right)\left[-\bar{\omega}_{i} \bar{\sigma}_{n-1}+\bar{\omega}_{n-1} \bar{\sigma}_{i}\right]\right. \\
\left.+\bar{\omega}_{i}\left[\rho_{n-1}-\beta_{n-1, n} \sin \left(\bar{\sigma}_{n-1}+\Delta_{n-1}^{s}\right)\right]\right) d t
\end{aligned}
$$

Noting that $\sigma_{i}=A_{k} \sin \left(\Omega_{i} t\right)+0(\alpha)$, first substitute in the Hamiltonian equation (6.1), then rewrite the Melnikov integral as

$$
\begin{aligned}
\tilde{\tilde{\boldsymbol{M}}}_{i}= & \int_{-\infty}^{\infty}\left\{G_{i}, H^{1}\right\} d t \\
= & \left(\beta _ { i n } \operatorname { c o s } ( \Delta _ { i } ^ { s } ) \left[\left(\bar{\sigma}_{n-1} A_{i} \cos \left(\Omega_{i} t\right)\right)_{-\infty}^{\infty} \cos \Omega_{i} t_{i}\right.\right. \\
& \left.+\left(\bar{\sigma}_{n-1} A_{i} \sin \left(\Omega_{i} t\right)\right)_{-\infty}^{\infty} \sin \Omega_{i} t_{i}\right] \\
& +\left[\int_{-\infty}^{\infty} V_{1}\left\{-\Omega_{i} A_{i} \sin \Omega_{i} t\right\} d t\right] \cos \left(\Omega_{i} t_{i}\right) \\
& -\left[\int_{\infty}^{\infty} V_{1}\left\{-\Omega_{i} A_{i} \cos \left(\Omega_{i}\right) t\right\} d t\right] \sin \left(\Omega_{i} t_{i}\right)
\end{aligned}
$$

where we have used

$$
\frac{d}{d t} \overline{\boldsymbol{\sigma}}_{i} \overline{\boldsymbol{\sigma}}_{n-1}=\bar{\omega}_{i} \overline{\boldsymbol{\sigma}}_{n-1}+\overline{\boldsymbol{\sigma}}_{i} \bar{\omega}_{n-1}
$$

and

$$
\begin{aligned}
V_{1}= & -2\left(\beta_{i n} \cos \left(\Delta_{i}^{s}\right) \bar{\sigma}_{n-1}+\rho_{n-1}\right. \\
& -\beta_{n-1, n} \sin \left(\bar{\sigma}_{n-1}+\Delta_{n-1}^{s}\right) .
\end{aligned}
$$

Similarly, one computes the following Melnikov integral

$$
\begin{aligned}
H_{\mu}^{1}= & \mu H^{1}=\alpha^{2} \sum_{i=1}^{n-2} \mu \beta_{i n} \cos \left(\Delta_{i}^{s}\right) \sigma_{i} \sum_{k=1}^{n-1} \sigma_{k}-\alpha \mu \bar{\rho}_{n-1} \sum_{k=1}^{n-1} \sigma_{k}+\alpha^{2} \mu \sum_{k=1}^{n-1} \sigma_{k} \beta_{n-1, n} \sin \left(\sigma_{n-1}+\Delta_{n-1}^{s}\right) \\
& +\frac{1}{2} \alpha^{2} \mu\left[\sum_{k=1}^{n-1} \omega_{k}\right]^{2}+0\left(\alpha^{1} \mu^{s}\right)
\end{aligned}
$$

where $s=2$ and $l=1$.

We now compute the Melnikov integrals along a homoclinic.orbit $\left(\bar{\omega}_{1}, \cdots, \bar{\omega}_{n-1}, \bar{\sigma}_{1}, \cdots, \bar{\sigma}_{n-1}\right)$.

$$
\begin{aligned}
\tilde{\tilde{M}}_{i}= & \int_{-\infty}^{\infty}\left\{G_{i}, H^{1}\right\} d t=\int_{-\infty}^{\infty}\left(\bar{\omega}_{i} \cdot\left[-\beta_{i n} \cos \left(\Delta_{i}^{s}\right) \sum_{k=1}^{n-1} \overline{\boldsymbol{\sigma}}_{k}\right]+\bar{\omega}_{i} \cdot\left[-\sum_{j=1}^{n-2} \beta_{j n} \cos \left(\Delta_{j}^{s}\right) \overline{\boldsymbol{\sigma}}_{j}\right]\right. \\
& \left.+\bar{\omega}_{i} \cdot\left[\bar{\rho}_{n-1}-\beta_{n-1, n} \sin \left(\overline{\boldsymbol{\sigma}}_{n-1}+\Delta_{n-1}^{s}\right)\right]+\left(\sum_{k=1}^{n-1} \bar{\omega}_{k}\right)\left(\beta_{\mathrm{in}} \cos \left(\Delta_{i}^{s}\right) \bar{\sigma}_{i}\right)\right) d t
\end{aligned}
$$




$$
\begin{aligned}
\tilde{\tilde{M}}_{n-1}=\int_{-\infty}^{\infty}\left\{F, H^{1}\right\} d t= & \int_{-\infty}^{\infty}\left(\overline { \omega } _ { n - 1 } \cdot \left[-\beta_{n-1, n} \cos \left(\bar{\sigma}_{n-1}+\Delta_{n-1}^{s}\right) \sum_{k=1}^{n-1} \bar{\sigma}_{k}-\sum_{j=1}^{n-2}\left(\beta_{j n} \cos \Delta_{j}^{s}\right) \bar{\sigma}_{j}\right.\right. \\
& +\left(\bar{\rho}_{n-1}-\beta_{n-1, n} \sin \left(\bar{\sigma}_{n-1}+\Delta_{n-1}^{s}\right)\right]-\left(\sum_{k=1}^{n-1} \bar{\omega}_{k}\right) \cdot\left(\bar{\rho}_{n-1}-\beta_{n-1, n} \sin \left(\bar{\sigma}_{n-1}+\Delta_{n-1}^{s}\right)\right) d t
\end{aligned}
$$

which, with $\rho_{n-1}:=\bar{\rho}_{n-1}$ and $\beta_{n-1}:=\beta_{n-1, n}$, reduces to (see Appendix A)

$$
\begin{aligned}
\tilde{\tilde{M}}_{n-1}= & \sum_{k=1}^{n-2} \int_{-\infty}^{\infty}\left(\frac{d}{d t}\left[\rho_{n-1}-\beta_{n-1} \sin \left(\bar{\sigma}_{n-1}+\Delta_{n-1}^{s}\right)\right] \bar{\sigma}_{k}\right. \\
& -\left[\rho_{n-1}-\beta_{n-1} \sin \left(\bar{\sigma}_{n-1}+\Delta_{n-1}^{s}\right)\right] \bar{\omega}_{k} \\
& \left.-\beta_{k n} \cos \left(\Delta_{k}^{s}\right) \bar{\omega}_{n-1} \bar{\sigma}_{k}\right) d t
\end{aligned}
$$

by direct computations and noting that $\sigma_{k}\left(t-t_{k}\right)=$ $A_{k} \cos \left(t-t_{k}\right)$, one obtains

$$
\begin{aligned}
\tilde{\tilde{M}}_{n-1}= & \sum_{k=1}^{n-2}\left(\left[\rho_{n-1}-\beta_{n-1} \sin \left(\bar{\sigma}_{n-1}+\Delta_{n-1}^{s}\right)\right]\right. \\
& \left.\cdot A_{k} \cos \Omega_{k} t\right)_{-\infty}^{\infty} \cos \Omega_{k} t_{k} \\
& +\left(\left[\rho_{n-1}-\beta_{n-1} \sin \left(\bar{\sigma}_{n-1}+\Delta_{n-1}^{s}\right)\right]\right. \\
& \left.\cdot A_{k} \sin \Omega_{k} t\right)_{-\infty}^{\infty} \sin \Omega_{k} t_{k} \\
& +2\left(\int_{-\infty}^{\infty}\left[\rho_{n-1}-\beta_{n-1} \sin \left(\bar{\sigma}_{n-1}+\Delta_{n-1}^{s}\right)\right]\right. \\
& \left.\cdot \Omega_{k} A_{k} \sin \Omega_{k} t d t\right) \cos \Omega_{k} t_{k} \\
& -2\left(\int_{-\infty}^{\infty}\left[\rho_{n-1}-\beta_{n-1} \sin \left(\bar{\sigma}_{n-1}+\Delta_{n-1}^{s}\right)\right]\right. \\
& \left.\cdot \Omega_{k} A_{k} \cos \Omega_{k} t d t\right) \sin \Omega_{k} t_{k} \\
& -\left(\int_{-\infty}^{\infty}\left(\beta_{k n} \cos \left(\Delta_{k}^{s}\right)\right) \bar{\omega}_{n-1} A_{k} \cos \left(\Omega_{k} t\right) d t\right) \\
& \cdot \cos \left(\Omega_{k} t_{k}\right) \\
& -\left(\int_{-\infty}^{\infty}\left(\beta_{k n} \cos \left(\Delta_{k}^{s}\right)\right) \bar{\omega}_{n-1} A_{k} \sin \left(\Omega_{k} t\right) d t\right) \\
& \cdot \sin \left(\Omega_{k} t_{k}\right) .
\end{aligned}
$$

From Appendix A and (6.3.i) one obtains

$$
\tilde{\tilde{M}}_{i}\left(t_{i}\right)=a_{i 1} \cos \Omega_{i} t_{i}+b_{i 1} \sin \Omega_{i} t_{i}
$$

where $a_{i 1}, b_{i 1}$ are nonzero constants for all except a discrete set of frequencies $\Omega_{i}$. Thus $\tilde{\boldsymbol{M}}_{i}$ has isolated zeros in $t_{i}$ (two in each period $2\left(\pi / \Omega_{i}\right)$ ). Also at these (isolated) discrete zeros, $\partial \tilde{\tilde{M}}_{i} / \partial t_{i} \neq 0$. Indeed, we set $\tilde{\boldsymbol{M}}_{i}=0$ in (6.6.i) to obtain

$$
\tan \left(\Omega_{i} t_{i}^{*}\right)=-\frac{a_{i 1}}{b_{i 1}}
$$

Let us define

$$
\sin \left(\Omega_{i} t_{i}^{*}\right)=-\frac{a_{i 1}}{\left[a_{i 1}^{2}+b_{i 1}^{2}\right]^{1 / 2}} .
$$

Equation (6.7.i) gives

Thus

$$
\Omega_{i} t_{i}^{*}=-\tan ^{-1}\left(\frac{a_{i 1}}{b_{i 1}}\right)+\bmod (\pi)
$$

$$
\begin{aligned}
& \frac{\partial \tilde{\tilde{M}}_{i}}{\partial t_{i}}\left(t_{i}^{*}\right)=-a_{i 1} \Omega_{i} \sin \Omega_{i} t_{i}^{*}+\Omega_{i} b_{i 1} \cos \Omega_{i} t_{i}^{*} \\
& \frac{\partial \tilde{\tilde{M}}_{i}}{\partial t_{i}}\left(t_{i}^{*}\right)=\Omega_{i}\left[\sqrt{a_{i 1}^{2}+b_{i 1}^{2}}\right] \neq 0 .
\end{aligned}
$$

Therefore, for almost all frequencies $\Omega_{i}, \tilde{\tilde{M}}_{i}\left(t_{i}^{*}\right)=0$ and $\partial \tilde{M}_{i} / \partial t_{i}\left(t_{i}^{*}\right) \neq 0$ on the set

$$
\left\{t_{i}^{*} \in R \mid(\text { eq. (6.8.i)) is satisfied }\}\right. \text {. }
$$

From Appendix A and (6.5) it follows that

$$
\begin{aligned}
\tilde{\tilde{M}}_{n-1}( & \left.t_{1}, \cdots, t_{n-3}, t_{n-2}\right) \\
& =\sum_{k=1}^{n-2} a_{n-1, k} \cos \left(\Omega_{k} t_{k}\right)+b_{n-1, k} \sin \left(\Omega_{k} t_{k}\right)
\end{aligned}
$$

where $a_{n-1, k}, b_{n-1, k}$ are nonzero for all $k, 1 \leqslant k \leqslant n-2$.

To obtain a transversal zero for the $(n-2)$-vector $\tilde{\tilde{M}}$ we observe that

$$
\tilde{\tilde{\boldsymbol{M}}}\left(t_{1}, \cdots, t_{n-2}\right)=\left(\tilde{\tilde{M}}_{1}, \cdots, \tilde{\tilde{\boldsymbol{M}}}_{n-3}, \tilde{\tilde{\boldsymbol{M}}}_{n-1}\right)^{t}
$$

has the Jacobian determinant

$$
\operatorname{det}[\boldsymbol{D} \tilde{\boldsymbol{M}}]=\prod_{k=1}^{n-3} \frac{\partial \tilde{\tilde{M}}_{k}}{\partial t_{k}} \cdot \frac{\partial \tilde{\tilde{M}}_{n-1}}{\partial t_{n-2}} .
$$

This follows since $\tilde{\tilde{\boldsymbol{M}}}_{k}$ is a function only of $t_{k}, k=1, \cdots, n$ -3 , hence the Jacobian matrix $\boldsymbol{D} \tilde{\boldsymbol{M}}$ is a lower triangular matrix. Thus if a transversal zero $t_{k}^{*}$ of each $\tilde{\boldsymbol{M}}_{k}, k=$ $1, \cdots, n-3$ is substituted into (5.10), then one would only require that the Melnikov function of (6.10) possess a transversal zero in the variable $t_{n-1}$. In this way, (6.11) will be trivially satisfied and it then follows that the vector $\tilde{\boldsymbol{M}}$ has a transversal zero.

To establish these claims, first substitute in (6.10) the transversal zeros $t_{1}^{*}, t_{2}^{*}, \cdots, t_{n-3}^{*}$ of $\tilde{\tilde{M}}_{k}, k=1, \cdots, n-3$ and obtain

$$
\begin{aligned}
\tilde{\tilde{M}}_{n-1}=a_{n-1, n-2} \cos \Omega_{n-2} t_{n-2} & +b_{n-1, n-2} \sin \Omega_{n-2} t_{n-2} \\
& +\sum_{k=1}^{n-3} \frac{a_{n-1, k} b_{k 1}-b_{n-1, k} a_{k 1}}{\left(a_{k 1}^{2}+b_{k 1}^{2}\right)^{1 / 2}}
\end{aligned}
$$

where we have used the following two equalities obtained from (6.7),

$$
\begin{aligned}
& \sin \left(\Omega_{k} t_{k}^{*}\right)=-\frac{a_{k 1}}{\left(a_{k 1}^{2}+b_{k 1}^{2}\right)^{1 / 2}} \\
& \cos \left(\Omega_{k} t_{k}^{*}\right)=+\frac{b_{k 1}}{\left(a_{k 1}^{2}+b_{k 1}^{2}\right)^{1 / 2}} .
\end{aligned}
$$


Thus for $\tilde{\tilde{M}}_{n-1}=0$, one requires

$$
\begin{aligned}
{\left[\begin{array}{ll}
a_{n-1, n-2} & b_{n-1, n-2}
\end{array}\right]\left[\begin{array}{c}
\cos \Omega_{n-2} t_{n-2} \\
\sin \left(\Omega_{n-2} t_{n-2}\right.
\end{array}\right] } \\
=\sum_{k=1}^{n-3}-\frac{a_{k 1} b_{n-1, k}-b_{k 1} a_{n-1, k}}{\left(a_{k 1}^{2}+b_{k 1}^{2}\right)^{1 / 2}} .
\end{aligned}
$$

Taking derivatives of (6.10) with respect to $t_{n-2}$,

$$
\frac{\partial \tilde{\tilde{M}}_{n-1}}{\partial t_{n-2}}=\Omega_{n-2}\left[\begin{array}{ll}
b_{n-1, n-2} & -a_{n-1, n-2}
\end{array}\right]\left[\begin{array}{c}
\cos \Omega_{n-2} t_{n-2} \\
\sin \Omega_{n-2} t_{n-2}
\end{array}\right] \text {. }
$$

To ensure that (6.13) does not vanish, we require that

$$
\left[\begin{array}{c}
\cos \Omega_{n-2} t_{n-2} \\
\sin \Omega_{n-2} t_{n-2}
\end{array}\right] \neq k_{1}\left[\begin{array}{l}
a_{n-1, n-2} \\
b_{n-1, n-2}
\end{array}\right]
$$

where $k_{1} \in R$, giving

\section{Theorem 6.1}

If (6.12) and (6.14) are satisfied for some $\left(t_{n-2}\right)$ then, for a sufficiently large $n$th machine $\left(M_{n}\right)$, Arnold diffusion arises in the Hamiltonian system (6.1) on every energy level $h>\bar{h}$ and $h$ is near $\bar{h}$.

We note that (6.12) and (6.14) are in fact satisfied by discrete values of $t_{n-2}$.

If one requires that the sum in (6.12) vanishes, one obtains the following condition:

$$
\left[\begin{array}{c}
\cos \Omega_{n-2} t_{n-2} \\
\sin \Omega_{n-2} t_{n-2}
\end{array}\right]=k_{2}\left[\begin{array}{c}
b_{n-1, n-2} \\
-a_{n-1, n-2}
\end{array}\right]
$$

where $k_{2}$ is a nonzero real constant. Then (6.13) becomes

$$
\frac{\partial \tilde{\tilde{M}}_{n-1}}{\partial t_{n-1}}=k_{2} \Omega_{n-2}\left[\left(b_{n-1, n-2}\right)^{2}+\left(a_{n-1, n-2}\right)^{2}\right] \neq 0 .
$$

Therefore, a sufficient condition for the Melnikov vector $\tilde{\tilde{\boldsymbol{M}}}$ to possess a transversal zero is

$$
\sum_{k=1}^{n-3} \frac{a_{k 1} b_{n-1, k}-b_{k 1} a_{n-1, k}}{\left(a_{k 1}^{2}+b_{k 1}^{2}\right)^{1 / 2}}=0
$$

or equivalently (using (6.12))

$$
\tan \left(\Omega_{n-2} t_{n-2}\right)=-\frac{a_{n-1, n-2}}{b_{n-1, n-2}} .
$$

We state the following:

Corollary: If (AA), (or (BB)), holds, then for sufficiently large machine $\left(M_{n}\right)$, Arnold diffusion arises in the Hamiltonian system (6.1) on infinitely many energy levels $h>\bar{h}$.

\section{CONCLUSIONS}

Theorems 5.1 and 6.1 can be extended to the case when the small parameter $\epsilon$ (see Section IV) is nonzero. This is possible as long as $\epsilon$ is in the order of $\mu^{2}$ (i.e., $0(\epsilon)=0\left(\mu^{2}\right)$ ) and thus it will have no effect on the existence of transversal zeros. This follows since the Melnikov method respects only the first terms of a perturbed solution expansion in a power series in $\mu$.

Our results ensure the presence of horseshoes (and hence chaotic orbits) in the special case of the two degree-of-freedom swing equations (three machines). This is similar to the case considered by Kopell and Washburn [29].

Holmes and Marsden [26] have developed a technique which may be employed to show that, on certain energy surfaces, Arnold diffusion can survive suitable positive and negative damping perturbations. It would be interesting to see if this technique can be applied in the case of the linear damping of the swing equations (i.e., $D_{i} \neq 0$ ).

We finally summarize our conclusions and suggestions for future work in the following.

(a) Theoretical Work:

(1) In Melnikov integrals for $n$ degree-of-freedom systems terms due only to products of oscillators may arise. These terms are not a measure of the separation between stable and unstable manifolds. They, rather, measure asynchronous distance between the oscillators. This phenomena, found by the present authors, needs to be explored more systematically.

(2) The Melnikov approach can be extended to consider more terms in the approximation of the separation between the stable and unstable manifolds. This seems appropriate from applications point of view since it is tuned for computations.

(3) Allowing for certain positive and negative damping in the theory of Arnold diffusion has been mentioned in Holmes and Marsden [25], [26]. The affects of damping needs to be explored for the case of the classical model of power systems.

(b) Applications:

(1) Many model systems in the physical sciences and engineering exist which satisfy the conditions of the theorems on chaotic behavior or Arnold diffusion. One needs to test for the presence of complicated dynamics of these models. For example, a study of the Josephson junction circuit (with negligible damping) can be conducted on lines similar to our approach.

(2) Computer simulations would verify the presence of complex irregular dynamics in the swing equations under the conditions provided in Section IV. Moreover they would display these dynamics for possible further studies. In the case of periodic but not necessarily small oscillators, tests can be conducted computationally.

(3) The effects of a small amount of damping should be studied. This allows for a more realistic modeling of many engineering systems.

(4) For large perturbations (large $\mu$ ) one can get many other effects, such as collision of nonresonant tori (see [1] and the references therein). The systematic exploration of these would be useful for many engineering systems in general and power systems specifically.

\section{APPENDIX A}

\section{ThE Simplification OF THE Melnikov INTEgRals For SMall Amplitude Oscillations}

We perform explicit calculations first to show that the integrals of a product of variables of oscillators over the infinite integral domain vanish. Second we show that integrals of products of oscillator variables with the homo- 
clinic orbit variables (subscripted $n-1)$ result in (6.6.i) and (6.10).

Equation (6.6.i) Case 1: Products of Oscillator Variables

From (6.2.i) we note that the products of oscillator variables are composed of the following basic integral.

$$
\Psi=\int_{-\infty}^{\infty} \bar{\omega}_{i}\left(t-t_{i}\right) \bar{\sigma}_{k}\left(t-t_{k}\right) d t
$$

where the bar denotes the unperturbed solutions given by

$$
\begin{aligned}
& \alpha \bar{\sigma}_{k}(t)=\alpha A_{k} \cos \Omega_{k} t+.0\left(\alpha^{2}\right) \\
& \alpha \bar{\omega}_{i}(t)=-\alpha A_{i} \Omega_{i} \sin \Omega_{i} t+0\left(\alpha^{2}\right)
\end{aligned}
$$

where $\alpha$ is sufficiently small. Perform a change of integration variables on (A.1) and obtain

$$
\Psi=\int_{-\infty}^{\infty} \bar{\omega}_{i}\left(\tau+t_{k}-t_{i}\right) \bar{\sigma}_{k}(\tau) d \tau .
$$

For $i=k, t_{i}=t_{k}$ and so

$$
\begin{aligned}
\Psi & =\int_{-\infty}^{\infty} \bar{\omega}_{k}(\tau) \bar{\sigma}_{k}(\tau) d \tau \\
& =\int_{-\infty}^{\infty}-A_{k}^{2} \Omega_{k} \sin \left(\Omega_{k} \tau\right) \cos \left(\Omega_{k} \tau\right) d \tau \\
& =C_{k} \int_{-\infty}^{\infty} \frac{1}{2} \sin \left(2 \Omega_{k} \tau\right) d \tau \\
& =\lim _{L \rightarrow \infty} \frac{C_{k}}{2}[\cos (L)-\cos (-L)]=0 .
\end{aligned}
$$

Note that we have substituted the first terms of the expressions for the unperturbed solutions. The error terms, $O\left(\alpha^{2}\right)$ are included in the higher order terms that do not affect the Melnikov integrals. To see this, first substitute the small oscillation variables in the Hamiltonian function and thus the $0\left(\alpha^{2}\right)$ term will be collected with the higher order terms. Now rewrite the Melnikov integrals.

For $k \neq i$, let $t_{i}^{*}=t_{k}-t_{i}$, then

$$
\begin{aligned}
\Psi= & \int_{-L}^{L}-A_{i} A_{k} \Omega_{i} \sin \Omega_{i}\left(\tau+t_{i}^{*}\right) \cos \left(\Omega_{k} \tau\right) d \tau \\
= & \frac{-A_{i} A_{k} \Omega_{i}}{2} \int_{-L}^{L}\left[\sin \left[\left(\Omega_{i}+\Omega_{k}\right) \tau+\Omega_{i} t_{i}^{*}\right]\right. \\
& \left.+\sin \left[\left(\Omega_{i}-\Omega_{k}\right) \tau+\Omega_{i} t_{i}^{*}\right]\right] d \tau .
\end{aligned}
$$

To get conditional convergence for a fixed $i$ and $k$, write

$$
\begin{aligned}
\Psi=A_{i} A_{k} \frac{\Omega_{i}}{2} \lim _{L \rightarrow \infty}\left\{\left.\frac{\cos \left[\left(\Omega_{i}+\Omega_{k}\right) \tau+\Omega_{i} t_{i}^{*}\right]}{\Omega_{i}+\Omega_{k}}\right|_{-L} ^{L}\right. \\
\left.+\left.\frac{\cos \left[\left(\Omega_{i}-\Omega_{k}\right) \tau+\Omega_{i} t_{i}^{*}\right]}{\Omega_{i}-\Omega_{k}}\right|_{-L} ^{L}\right\} .
\end{aligned}
$$

Let

$$
\begin{aligned}
& \Psi_{1}=\left.\frac{\cos \left[\left(\Omega_{i}+\Omega_{k}\right) \tau+\Omega_{i} t_{i}^{*}\right]}{\Omega_{i}+\Omega_{k}}\right|_{-L} ^{L} \\
& \Psi_{2}=\left.\frac{\cos \left[\left(\Omega_{i}-\Omega_{k}\right) \tau+\Omega_{i} t_{i}^{*}\right]}{\Omega_{i}-\Omega_{k}}\right|_{-L} ^{L} .
\end{aligned}
$$

We claim that there is a sequence $L_{n}$ of values of $L$ converging to $\infty$ such that $\Psi=0$. To see this, write

$$
\begin{aligned}
\Psi_{1}= & \frac{1}{\left(\Omega_{i}+\Omega_{k}\right)}\left\{\cos \left[\left(\Omega_{i}+\Omega_{k}\right) L\right] \cos \Omega_{i} t_{i}^{*}\right. \\
& -\sin \left[\left(\Omega_{i}+\Omega_{k}\right)\right] \sin \Omega_{i} t_{i}^{*} \\
& -\left(\cos \left[-\left(\Omega_{i}+\Omega_{k}\right) L\right] \cos \Omega_{i} t_{i}^{*}\right. \\
& \left.\left.-\sin \left[-\left(\Omega_{i}+\Omega_{k}\right) L\right] \sin \Omega_{i} t_{i}^{*}\right)\right\} \\
= & \frac{1}{\left(\Omega_{i}+\Omega_{k}\right)}\left\{-2 \sin \left[\left(\Omega_{i}+\Omega_{k}\right) L\right] \sin \Omega_{i} t_{i}^{*}\right\} .
\end{aligned}
$$

Similarly,

$$
\begin{aligned}
\Psi_{2}= & \frac{1}{\left(\Omega_{i}-\Omega_{k}\right)}\left\{\cos \left[\left(\Omega_{i}-\Omega_{k}\right) L\right] \cos \Omega_{i} t_{i}^{*}\right. \\
& -\sin \left[\left(\Omega_{i}-\Omega_{k}\right)\right] \sin \Omega_{i} t_{i}^{*} \\
& -\left(\cos \left[-\left(\Omega_{i}-\Omega_{k}\right) L\right] \cos \Omega_{i} t_{i}^{*}\right. \\
& \left.\left.-\sin \left[-\left(\Omega_{i}-\Omega_{k}\right) L\right] \sin \Omega_{i} t_{i}^{*}\right)\right\} \\
= & \frac{1}{\left(\Omega_{i}-\Omega_{k}\right)}\left\{-2 \sin \left[\left(\Omega_{i}-\Omega_{k}\right) L\right] \sin \Omega_{i} t_{i}^{*}\right\} .
\end{aligned}
$$

Then consider

$$
\begin{aligned}
\bar{\Psi}= & \left(\Omega_{i}+\Omega_{k}\right)\left(\Omega_{i}-\Omega_{k}\right) \Psi \\
= & A\left\{\left(\Omega_{i}-\Omega_{k}\right) \sin \left[\left(\Omega_{i}+\Omega_{k}\right) L\right]\right. \\
& \left.+\left(\Omega_{i}+\Omega_{k}\right) \sin \left[\left(\Omega_{i}-\Omega_{k}\right) L\right]\right\} \sin \Omega_{i} t_{i}^{*} \\
\bar{\Psi}= & A \sin \Omega_{i} t_{i}^{*}\left\{\left(\Omega_{i}-\Omega_{k}\right)\right. \\
& \cdot\left[\sin \Omega_{i} L \cos \Omega_{k} L+\cos \Omega_{i} L \sin \Omega_{k} L\right] \\
& \left.+\left(\Omega_{i}+\Omega_{k}\right)\left[\sin \Omega_{i} L \cos \Omega_{k} L-\cos \Omega_{i} L \sin \Omega_{k} L\right]\right\} \\
\bar{\Psi}= & A \sin \Omega_{i} t_{i}^{*}\left\{\sin \Omega_{i} L \cos \Omega_{k} L\left(\Omega_{i}-\Omega_{k}+\Omega_{i}+\Omega_{k}\right)\right. \\
& \left.+\cos \Omega_{i} L \sin \Omega_{k} L\left(\Omega_{i}-\Omega_{k}-\Omega_{i}-\Omega_{k}\right)\right\} \\
= & 2 A \sin \Omega_{i} t_{i}^{*} \\
& \cdot\left\{\Omega_{i}\left(\sin \Omega_{i} L \cos \Omega_{k} L\right)-\Omega_{k}\left(\cos \Omega_{i} L \sin \Omega_{k} L\right)\right\} .
\end{aligned}
$$

Consider the quantity in braces

$$
\left\{\Omega_{i} \sin \Omega_{i} L \cos \Omega_{k} L-\Omega_{k} \cos \Omega_{i} L \sin \Omega_{k} L\right\}
$$

which we equate with zero and obtain

$$
\tan \left(\Omega_{i} L\right)=\frac{\Omega_{k}}{\Omega_{i}} \tan \left(\Omega_{k} L\right) .
$$

A simple sketch of these functions shows that there exist a sequence $L_{n}$ of time, $L_{n} \rightarrow \infty$, values at which the equality holds. (Rational independence of the frequencies is sufficient for the existence of this sequence.)

Case 2: A product of a homoclinic and oscillator variables

The basic product components of the Melnikov integral (6.2.i) are as follows:

$$
\begin{aligned}
& \int_{-\infty}^{\infty} \bar{\omega}_{i}\left(\tau-t_{i}\right) \bar{\sigma}_{n-1}(\tau) d \tau \\
& \int_{-\infty}^{\infty} \bar{\omega}_{n-1}(\tau) \bar{\sigma}_{i}\left(\tau-t_{i}\right) d \tau \\
& \int_{-\infty}^{\infty} \bar{\omega}_{i}\left(\tau-t_{i}\right) \dot{\bar{\omega}}_{n-1}(\tau) d \tau
\end{aligned}
$$


where $t_{i}$ is the initial phase.

$$
\begin{aligned}
& \int_{-\infty}^{\infty} \bar{\omega}_{i}\left(\tau-t_{i}\right) \dot{\bar{\omega}}_{n-1}(\tau) d \tau \\
& \quad=\lim _{L \rightarrow \infty}\left\{\left[\bar{\omega}_{i}\left(\tau-t_{i}\right) \bar{\omega}_{n-1}(\tau)\right]_{-L}^{L}-\int_{-L}^{L} \bar{\omega}_{i} \bar{\omega}_{n-1} d \tau\right\} .
\end{aligned}
$$

Noting that $\lim _{L \rightarrow \infty} \bar{\omega}_{n-1}( \pm L)=0$ (the velocity component of the saddle of the homoclinic orbit equals zero), then

$$
\begin{aligned}
\int_{-\infty}^{\infty} \bar{\omega}_{i}\left(\tau-t_{i}\right) \dot{\bar{\omega}}_{n-1}(\tau) d \tau \\
=\lim _{L \rightarrow \infty}\left\{-\int_{-L}^{L} \dot{\bar{\omega}}_{i} \bar{\omega}_{n-1} d \tau\right\} \\
=\lim _{L \rightarrow \infty}-\left\{\left[\dot{\bar{\omega}}_{i}\left(\tau-t_{i}^{*}\right) \bar{\sigma}_{n-1}(\tau)\right]_{-L}^{L}\right. \\
\\
\left.-\int_{-L}^{L} \frac{d}{d \tau} \dot{\bar{\omega}}_{i}\left(\tau-t_{i}^{*}\right) \bar{\sigma}_{n-1}(\tau) d \tau\right\}
\end{aligned}
$$

Again the first limit goes to zero. First note that

$$
\begin{aligned}
\bar{\omega}_{i}\left(\tau-t_{i}^{*}\right) & =-A_{i} \Omega_{i} \sin \left(\Omega_{i}\left(\tau-t_{i}^{*}\right)\right) \\
& =\bar{\omega}_{i}(\tau) \cos \left(\Omega_{i} t_{i}^{*}\right)+\Omega_{i} \bar{\sigma}_{i}(\tau) \sin \Omega_{i} t_{i}^{*}
\end{aligned}
$$

and

$$
\begin{aligned}
\bar{\sigma}_{i}\left(\tau-t_{i}^{*}\right) & =A_{i} \cos \left(\Omega_{i}\left(\tau-t_{i}^{*}\right)\right) \\
& =\left[\bar{\sigma}_{i}(\tau) \cos \left(\Omega_{i} t_{i}^{*}\right)+\frac{1}{\Omega_{i}} \bar{\omega}_{i}(\tau) \sin \left(\Omega_{i} t_{i}^{*}\right)\right]
\end{aligned}
$$

where we neglected the error terms which do not affect the Melnikov integrals. Then the first term of (A.2c) becomes

$$
\begin{aligned}
&\left.\lim _{L \rightarrow \infty} \bar{\sigma}_{n-1}(\tau) \dot{\bar{\omega}}_{i}\left(\tau-t_{i}^{*}\right)\right|_{-L} ^{L} \\
&=-\left.\Omega_{i}^{2} \lim _{M \rightarrow \infty} \bar{\sigma}_{n-1}(\tau) \bar{\sigma}_{i}\left(\tau-t_{i}^{*}\right)\right|_{\left(-M+t_{i}^{*}\right) / \Omega_{i}} ^{\left(M+t_{i}^{*}\right) / \Omega_{i}} \\
&=\lim _{M \rightarrow \infty} A_{i}\left\{\cos (M) \bar{\sigma}_{n-1}\left(\frac{M+t_{i}^{*}}{\Omega_{i}}\right)\right. \\
& \\
&\left.-\bar{\sigma}_{n-1}\left(\frac{-M+t_{i}^{*}}{\Omega_{i}}\right) \cos (-M)\right\} \\
&=\lim _{M \rightarrow \infty} A_{i} A_{i}\left[\bar{\sigma}_{n-1}\left(\frac{M+t_{i}^{*}}{\Omega_{i}}\right)\right. \\
&\left.-\bar{\sigma}_{n-1}\left(\frac{-M+t_{i}^{*}}{\Omega_{i}}\right)\right] \cos M
\end{aligned}
$$

where we wrote $A_{i} \cos \Omega_{i} \tau$ for $\bar{\sigma}_{i}(\tau)$. Note that $\bar{\sigma}_{n-1}$ is the component of the homoclinic orbit such that

$$
\lim _{M \rightarrow \infty} \bar{\sigma}_{n-1}( \pm M)=\bar{\sigma}_{n-1}( \pm \infty)=\left(\pi-2 \Delta_{n-1}^{s}\right)
$$

where the saddle point is $\left(\pi-2 \Delta_{n-1}^{s}, 0\right)$, and hence (A.3) vanishes. Therefore,

$$
\begin{aligned}
\int_{-\infty}^{\infty} \bar{\omega}_{i}\left(\tau-t_{i}\right) \dot{\bar{\omega}}_{n-1}(\tau) d \tau \\
=\int_{-\infty}^{\infty} \frac{d}{d \tau} \dot{\bar{\omega}}_{i}\left(\tau-t_{i}\right) \bar{\sigma}_{n-1}(\tau) d \tau \\
=-\Omega_{i}^{2} \int_{-\infty}^{\infty} \bar{\omega}_{i}\left(\tau-t_{i}\right) \bar{\sigma}_{n-1}(\tau) d \tau \\
=-\Omega_{i}^{2} \int_{-\infty}^{\infty} \bar{\omega}_{i}\left(\tau-t_{i}\right) \bar{\sigma}_{n-1}(\tau) d \tau
\end{aligned}
$$

Thus the basic terms are those of (A.2a) and (A.2b).

The integral of (A.2b) is treated the same way,

$$
\begin{aligned}
\int_{-\infty}^{\infty} \bar{\omega}_{n-1}(\tau) \bar{\sigma}_{i}\left(\tau-t_{i}^{*}\right) d \tau & \\
= & \left.\lim _{N \rightarrow \infty} \bar{\sigma}_{n-1}(\tau) \bar{\sigma}_{i}\left(\tau-t_{i}^{*}\right)\right|_{-N / \Omega_{i}} ^{N / \Omega_{i}} \\
& -\int_{-\infty}^{\infty} \bar{\sigma}_{n-1}(\tau) \bar{\omega}_{i}\left(\tau-t_{i}^{*}\right) d \tau
\end{aligned}
$$

where the first term vanishes (see A.3) and hence (A.4) reduces to (the negative of) the integral in (A.2a). Thus

$$
\begin{aligned}
(\mathrm{A} .2 \mathrm{a})= & \int_{-\infty}^{\infty} \bar{\omega}_{i}\left(\tau-t_{i}^{*}\right) \bar{\sigma}_{n-1}(\tau) d \tau \\
= & \left\{\int_{-\infty}^{\infty} \bar{\sigma}_{n-1}(\tau) \bar{\omega}_{i}(\tau) d \tau\right\} \cos \Omega_{i} t_{i}^{*} \\
& +\left\{\Omega_{i} \int_{-\infty}^{\infty} \bar{\sigma}_{n-1}(\tau) \bar{\sigma}_{i}(\tau) d \tau\right\} \sin \Omega_{i} t_{i}^{*}
\end{aligned}
$$

where each integral in the braces is well-defined and is a nonzero constant for all except a discrete set of $\Omega_{i}$ (see Kopell and Washburn [29] or Holmes and Marsden [27]).

Collecting terms and noting that the coefficients of these integrals are different due to the different parameters $M_{j}, B_{j}$, etc., one obtains the following:

$$
\tilde{\tilde{M}}_{i}\left(t_{i}\right)=a_{i 1} \cos \Omega_{i} t_{i}^{*}+b_{i 1} \sin \Omega_{i} t_{i}^{*}
$$

where $a_{i 1}$ and $b_{i 1}$ are nonzero constants for all except a discrete set of $\Omega_{i}$.

Equation (6.10):

The terms of the integrand of (6.4) are composed of the following expressions:

$$
\begin{array}{rr}
\frac{d}{d t}\left[\rho_{n-1}-\beta_{n-1, n} \sin \left(\bar{\sigma}_{n-1}+\Delta_{n-1}^{s}\right)\right] \bar{\sigma}_{n-1} & \text { (A.5.a) } \\
\frac{d}{d t}\left[\rho_{n-1}-\beta_{n-1, n} \sin \left(\bar{\sigma}_{n-1}+\Delta_{n-1}^{s}\right)\right] \bar{\sigma}_{k}, & 1 \leqslant k \leqslant n-2 \\
\bar{\omega}_{n-1} \bar{\sigma}_{k}, \quad 1 \leqslant k \leqslant n-2 & (\text { A.5.b) } \\
{\left[\rho_{n-1}-\beta_{n-1, n} \sin \left(\bar{\sigma}_{n-1}+\Delta_{n-1}^{s}\right)\right] \bar{\omega}_{n-1}} & (\text { A.5.c) } \\
{\left[\rho_{n-1}-\beta_{n-1, n} \sin \left(\bar{\sigma}_{n-1}+\Delta_{n-1}^{s}\right)\right] \bar{\omega}_{k}=\left(\dot{\bar{\omega}}_{n-1}\right) \bar{\omega}_{k} .}
\end{array}
$$

We first consider the terms with vanishing integrals over the infinite domains; namely (A.5.a) and (A.5.d). 
Consider the integral of (A.5.a) and perform integration and by parts.

$$
\begin{aligned}
& \int_{-\infty}^{\infty} \frac{d}{d t}\left[\rho_{n-1}-\beta_{n-1, n} \sin \left(\bar{\sigma}_{n-1}+\Delta_{n-1}^{s}\right)\right] \bar{\sigma}_{n-1} d \tau \\
& =\lim _{N \rightarrow \infty}\left\{\left.\bar{\sigma}_{n-1}(\tau)\left[\rho_{n-1}-\beta_{n-1, n} \sin \left(\bar{\sigma}_{n-1}(\tau)+\Delta_{n-1}^{s}\right)\right]\right|_{-N} ^{N}\right. \\
& \left.\quad-\int_{-L}^{L}\left[\rho_{n-1}-\beta_{n-1, n} \sin \left(\bar{\sigma}_{n-1}(\tau)+\Delta_{n-1}^{s}\right)\right] \bar{\omega}_{n-1}(\tau) d \tau\right\} .
\end{aligned}
$$

The first term vanishes as $N \rightarrow \infty$ since

$$
\begin{aligned}
\lim _{N \rightarrow \infty}\left[\rho_{n-1}-\beta_{n-1, n} \sin \left(\bar{\sigma}_{n-1}(N)+\Delta_{n-1}^{s}\right)\right] & \\
& =\lim _{N \rightarrow \infty} \dot{\bar{\omega}}_{n-1}(N)=0
\end{aligned}
$$

where $\bar{\omega}_{n-1}( \pm \infty)$ is a component of the saddle

$$
\left(\bar{\sigma}_{n-1}( \pm \infty), \bar{\omega}_{n-1}( \pm \infty)\right)=\left(\bar{\sigma}_{n-1}( \pm \infty), 0\right) .
$$

Thus the term of (A.5.a) is the same (except for a minus sign) as the term of (A.5.d). Consider now the integral of (A.5.d)

$$
\begin{aligned}
& \int_{-\infty}^{\infty} {\left[\rho_{n-1}-\beta_{n-1, n} \sin \left(\bar{\sigma}_{n-1}+\Delta_{n-1}^{s}\right)\right] \bar{\omega}_{n-1}(\tau) d \tau } \\
& \quad=\int_{-\infty}^{\infty} \dot{\bar{\omega}}_{n-1}(\tau) \bar{\omega}_{n-1}(\tau) d \tau=\left.\frac{1}{2} \lim _{N \rightarrow \infty} \bar{\omega}_{n-1}^{2}(\tau)\right|_{-N} ^{N}=0 .
\end{aligned}
$$

That is, the "kinetic" energy at the saddle, referenced to itself, is equals to zero. Hence the terms of (A.5.a) and (A.5.d) produce vanishing integrals.

The term of (A.5.c) is the same as the one in (A.4). The terms of (A.5.b) and (A.5.e) are the same if one performs integration by parts on (A.5.e).

Thus it is left only to consider the term (A.5.b) for a given $k, 1 \leqslant k \leqslant n-2$,

$$
\begin{aligned}
& \int_{-\infty}^{\infty}\left(\frac{d}{d t}\left[\rho_{n-1}-\beta_{n-1, n} \sin \left(\bar{\sigma}_{n-1}+\Delta_{n-1}^{s}\right)\right] \bar{\sigma}_{k}\right) d \tau \\
& =\int_{-\infty}^{\infty} \frac{d}{d t}\left[\rho_{n-1}-\beta_{n-1, n} \sin \left(\bar{\sigma}_{n-1}+\Delta_{n-1}^{s}\right)\right] \bar{\sigma}_{k}\left(t-t_{k}\right) d t \\
& =\int_{-\infty}^{\infty} \frac{d}{d t}\left[\dot{\bar{\omega}}_{n-1}\right] \bar{\sigma}_{k}\left(t-t_{k}\right) d t, \quad 1 \leqslant k \leqslant n-2 .
\end{aligned}
$$

Noting that

$$
\begin{aligned}
\bar{\sigma}_{k}\left(t-t_{k}\right) & =A_{k} \cos \Omega_{k}\left(t-t_{k}\right) \\
& =A_{k}\left[\cos \Omega_{k} t_{k} \cos \Omega_{k} t_{k}+\sin \Omega_{k} \sin \Omega_{k} t_{k}\right]
\end{aligned}
$$

then the integral becomes

$$
\begin{aligned}
\int_{-\infty}^{\infty}(\text { A.5.b }) d \tau= & {\left[A_{k} \int_{-\infty}^{\infty} \frac{d}{d t}\left(\dot{\bar{\omega}}_{n-1}\right) \cos \Omega_{k} t d t\right] \cos \Omega_{k} t_{k} } \\
& +\left[A_{k} \int_{-\infty}^{\infty} \frac{d}{d t}\left(\dot{\bar{\omega}}_{n-1}\right) \sin \Omega_{k} t d t\right] \sin \Omega_{k} t_{k} .
\end{aligned}
$$

We note that no cancellations between the integral terms in (6.4) can occur since the coefficients of the integrals are different for different parameters. Define

$$
a_{n-1, k}=A_{k} \int_{-\infty}^{\infty} \frac{d}{d t}\left(\dot{\bar{\omega}}_{n-1}(t)\right) \cos \Omega_{k} t d t
$$

$$
b_{n-1, k}=A_{k} \int_{-\infty}^{\infty} \frac{d}{d t}\left(\dot{\bar{\omega}}_{n-1}(t)\right) \sin \Omega_{k} t d t
$$

where $a_{n-1, k}$ and $b_{n-1, k}$ are well defined and vanish only for a set of discrete values of $\Omega_{i}$. This follows from the analyticity of the integrals in $\Omega_{k}$ with the rate of acceleration term (i.e., $\left.(d / d t) \dot{\bar{\omega}}_{n-1}(t)\right)$ is nonvanishing along the homoclinic orbit (scc Kopell and Washburn [29] or Holmes and Marsden [27]).

\section{ACKNOWLEDGMENT}

The authors thank Philip Holmes and Nancy Kopell for inspiration. They also thank Felix Wu, Eyad Abed, Aris Arapostathis, Rong Chen, Shankar Sastry, and Nikos Tsolas for valuable discussions.

\section{REFERENCES}

[1] F. M. Salam, J. Marsden, and P. Varaiya "Chaos and Arnold diffusion in dynamical systems," IEEE Circuits Syst., Nov. 1983.

[2] F. Abdel Salam, "Chaos and Arnold diffusion in dynamical systems with applications to power systems," Ph.D. dissertation, UC Berkeley, Dec. 1982.

[3] F. Abdel Salam, "Analytic Expressions for the unstable manifold of dynamical systems of differential equations," Masters thesis, UCB Dec. 1982.

[4] E. Abed and P. Varaiya, "Oscillations in power systems via Hopf bifurcation," Proc. of the 20th IEEE C. D.C., Dec. 1981, San Diego, CA.

[5] R. Abraham and J. E. Marsden, Foundations of Mechanics. 2nd ed., Reading, MA: Benjamin, 1978.

[6] P. M. Anderson and A. A. Fouad, Power System Control and Stability, Volume 1. Ames, IO: The Iowa State Univ. Press, 1977.

[7] A. A. Andronov and C. E. Chaikin, Theory of Oscillations. Princeton, NJ.: Princeton Univ. Press, 1949.

[8] A. Arapostathis, S. Sastry, and P. Varaiya, "Analysis of the power flow equations," Electrical Energy and Power Systems, vol. 3(3), $1981,116-126$.

[9] , "Global Analysis of swing dynamics," IEEE Trans. Circuits Syst., Oct. 1982.

[10] A. Arapostathis and P. Varaiya, "The behavior of three node power networks," Electric Energy and Power Systems, Jan. 1983.

[11] V. I. Arnold, "Instability of dynamical systems with several degrees of freedom," Dokl. Akad. Nauk. SSSR 156:9-12, 1964.

[12] _. Mathematical Methods of Classical Mechanics. BerlinHeidelberg: Springer-Verlag, 1978.

[1.3] T. Athay, R. Podmore, and S. Virmani, "A practical method for the direct analysis of transient stability," IEEE Trans. Power App. Syst., vol. PAS-98, pp. 573-584, 1979.

[14] T. Athay, V. Sherkat, R. Podmore, S. Virmani, and C. Puegh, "Transient Energy Stability Analysis," Systems Engineering for Power Conference, Davos, Switzerland, October 1979.

[15] P. D. Aylett, "The Energy Integral Criterion of Transient Stability Limits of Power Systems," Proc. IEE, vol. 105(C), pp. 527-536, 1958.

[16] J. Bailleul and C. I. Byrnes, "Geometric Critical Point Analysis of Lossless Power System Models," IEEE Trans. Circuits and Systems, vol. CAS-29, pp. 724-737, Nov. 1982.

[17] R. W. Brockett, "On the Asymptotic Properties of Solutions on differential Equations with Multiple Equilibria," Journal of Differential Equations, Vol. 44, number 2, may 1982, pp. 249-262.

[18] A. H. El-Abiad and K. Nagappan, "Transient Stability Regions of Multimachine Power Systems," IEEE Trans. Power App. Syst., vol. PAS-85, pp. 169-179, 1966

[19] A. A. Fouad, "Stability Theory-Criteria for Transient Stability," in Systems Engineering of Power: Status and Prospects (Ed. L. H. Fink and K. Carlson), U.S. ERDA and ERPI, 1975, pp. 421-450.

[20] A. A. Fouad and S. E. Stanton, "Transient Stability of a Multimachine Power System, Part I: Investigation of System Trajectories; Part II: Critical Transient Energy," IEEE Trans. Power App. Syst., vol. PAS-100, pp. 3408-3424, 1981.

[21] . U. Gudaru, "A general Lyapunov function for multi-machine power systems with transfer conductances," Int. J. Contr., 1975, vol. 21, no. 2, pp. 333-343. 
[22] C. L. Gupta and A. H. El-Abiad, "Determination of the Closest Unstable Equilibrium State for Lyapunov Methods in Transient Stability Studies," IEEE Trans. Power App. Syst., vol. PAS-94, pp. 1699-1712, 1976.

[23] D. J. Hill and A. R. Bergen, "Stability Analysis of Multimachine Power Networks with Linear Frequency Dependent Loads," Memo. UCB/ERL M81/5, Electronics Research Lab., Univ. of California, Berkeley, CA, Dec. 1980.

[24] P. Holmes, "Averaging and chaotic motions in forced oscillations," SIAM. J. Appl. Math., vol. 38, 68-80 and 40, 167-168, (1980).

[25] P. J. Holmes and J. E. Marsden, "Horseshoes in perturbations of Hamiltonian systems with two degrees of freedom" Commun. Math. Phys., vol. 82, 1982, pp. 523-544.

[26] , "Melnikov's method and Arnold diffusion for perturbations of integrable Hamiltonian systems," J. Math. Phys., vol. 23 no. 4, pp. $669-679,1982$

[27] "Horseshoes and Arnold diffusion for Hamiltonian systems on Lie groups," Indiana Univ. Math. J., Mar./Apr. 1983.

[28] Kakimoto, N., Ohsawa, Y., Hayashi, M., "Transient Stability Analysis of Electric Power Systems via Lure' Type Lyapunov Function." "Part I, New Critical Value for Transient Stability," pp. 62-71. "Part II. Modification of Lurc' Typc Lyapunov Function with Effect of Transfer Conductances," pp. 72-79. Trans. IEE Japan, vol. 98, no. 516, May/June 1978.

[29] N. Kopell and R. B. Washburn, Jr., "Chaotic motions in the two degree-of-freedom swing equations," IEEE Trans. Circuits Syst., Nov. 1982 , pp. $738-746$.

[30] A. Korsak, "On the Question of Uniqueness of Stable Load-Flow Solutions," IEEE Trans. Power App. Systems, vol. PAS-91 (1972), pp. $1033-1100$.

[31] G. A. Luders, "Transient stability of multimachine power systems via the direct method of Lyapunov," IEEE Trans. Power App. Syst., vol. PAS-90 pp. 23-36, 1971.

[32] M. A. Pai and P. G. Murthy, "On Lyapunov Functions for Power Systems with Transfer Conductances," IEEE Trans. Automatic Control, vol. AC-18, pp. 181-183, Apr. 1973.

[33] M. A. Pai, "Some mathematical aspects of power system stability by Lyapunov's method," Int. Conf. "Electric Power Problems: The mathematical chalenge," sponsored by SIAM, Seattle, WA, Mar. $18-20,1980$

[34] Prabhakara, F. S. and A. H. El-Abiad, "A simplified determination of transient stability regions for Lyapunov methods," IEEE Trans. Power App. Syst., vol. PAS-94, pp. 672-689, 1975.

[35] M. Ribbens-Pavella, "Critical survey of transient stability studies of multimachine power systems by Lyapunov's direct method," in Proc. 9th Allerton Conf. Circuit and System Theory, October 6-8, 1971, pp. 751-761.

[36] "Comments on J. L. Willems "Direct methods for transient stability studies in power system analysis," and Reply by Author, IEEE Trans. Automatic Control, vol. AC-17, pp. 415-417, June 1972.

[37] M. Ribbens-Pavella, A. Calvaer, and J. Gheury, "Transient stability index for on-line evaluation," IEEE PES Winter Meeting, New York, Fcb. 3-8, 1980.

[38] S. J. Skar, "Stability of multi-machine Power Systems with Nontrivial Transfer Conductances," SIAM J. Appl. Math., vol. 39, no. 3, December 1980.

[39] C. J. Tavora and O. J. M. Smith, "Equilibrium analysis of power systems," IEEE Trans. Power App. Syst., vol. PAS-91, pp. $1131-1137,1972$

[40] C. J. Tavora, and O. J. M. Smith, "Stability analysis of Power Systems," IEEE Trans. Power App. Syst., vol. PAS-91, pp. $1138-1144,1972$

[41] J. E. Van Ness, F. M. Brasch, Jr., G. L. Landgren and S. I. Neumann, "Analytical investigation of dynamic instability occuring at powerton station," IEEE Trans. Power App. Syst., vol. PAS-99, pp. 1386-1395, 1980.

[42] J. L. Willems, "The computation of finite stability regions by means of open Lyapunov surfaces," Int. J. Control, 1969, vol. 10, no. 5, pp. 537-544.

[43] $\longrightarrow$ "Direct methods for transient stability studies in power system analysis," IEEE Trans. Automatic Control, vol. AC-16, no. 4, August 1971

[44] "A partial stability approach to the problem of transient power system stability," Int. J. Control, 1974 , vol. 19 , no. 1 , pp. $1-14$.

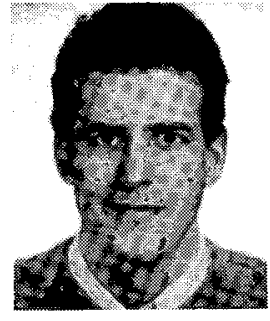

Fathi M. A. Salam received the M.S. degree in electrical engineering from the University of California, Davis, in 1979, the B.S. and Ph.D. degrees in 1976 and 1982, respectively, both in electrical engineering, and the M.A. degree in mathematics in 1982, from the University of California, Berkeley.

From 1976 to 1977 he worked for a subsidiary of Exxon in the instrumentation and maintenance division. He was a Visiting Assistant Professor of electrical engineering at the University of California at Berkeley, and is currently an Assistant Professor of Systems at Drexel University in the Department of Mechanical Engineering and Mechanics. His areas of research interest are linear and nonlinear systems, computational algorithms, complicated dynamics of chaos and Arnold diffusion, stability and control of power systems, and interconnected large scale systems analysis.

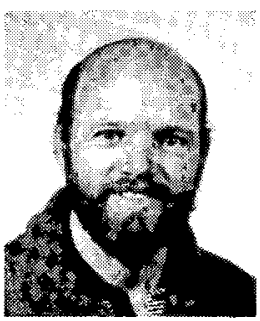

Jerrold E. Marsden is currently a Professor of Mathematics at the University of California, Berkeley, has also taught in Canada, France, and Britain. He holds the B.Sc. degree from the University of Toronto and the Ph.D. degree in Mathematical Physics from Princeton University. Dr. Marsden received the Gravitation Research Prize in 1973, a Carnegie Fellowship in 1977, a Killam Fellowship in 1979 and held a Miller Professorship in 1981-1982. Dr. Marsden's publications include the following books "Applications of Global Analysis in Mathematical Physics" (1974), "The Hopf Bifurcation and its Applications" (with M. McCracken, 1976), "Foundations of Mechanics" (with R. Abraham, 1978), "A Mathematical Introduction to Fluid Mechanics" (with A. Chorin, 1979), "The Mathematical Foundations of Elasticity" (with T. Hughes, 1983), and "Manifolds, Tensor Analysis and Applications" (with R. Abraham and T. Ratiu, 1983).

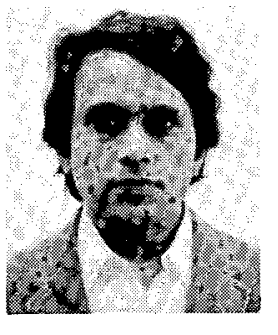

Pravin P. Varaiya (M'68-SM'78-F'80) received the B.E. degree from the University of Bombay, Bombay, India, in 1960, and the Ph.D. degree from the University of California, Berkeley, in 1966.

Since 1966 , he has been with the University of California, Berkeley, where he is currently Professor of Electrical Engineering and Computer Sciences. His research interests are in the areas of stochastic systems, large-scale systems, and urban economics. 